OPEN ACCESS

Edited by:

Dong Sun,

Virginia Commonwealth University,

United States

Reviewed by:

Maria Eugenia Caligiuri,

Università degli Studi Magna Graecia

di Catanzaro, Italy

Nataliya G. Kolosova,

Institute of Cytology and Genetics

(RAS), Russia

${ }^{*}$ Correspondence:

Rilkka Immonen

riikka.immonen@uef.fi

Specialty section:

This article was submitted to

Neurodegeneration,

a section of the journal

Frontiers in Neuroscience

Received: 09 May 2019

Accepted: 31 July 2019

Published: 16 August 2019

Citation:

Yasmin A, Pitkänen A, Jokivarsi K,

Poutiainen P, Gröhn $O$ and Immonen $R$ (2019) MRS Reveals

Chronic Inflammation in T2W

MRI-Negative Perilesional Cortex A 6-Months Multimodal Imaging

Follow-Up Study.

Front. Neurosci. 13:863.

doi: 10.3389/fnins.2019.00863

\section{MRS Reveals Chronic Inflammation in T2w MRI-Negative Perilesional Cortex - A 6-Months Multimodal Imaging Follow-Up Study}

\author{
Amna Yasmin'1, Asla Pitkänen ${ }^{1}$, Kimmo Jokivarsi' ${ }^{1}$, Pekka Poutiainen ${ }^{2}$, Olli Gröhn ${ }^{1}$ and \\ Riikka Immonen ${ }^{1 *}$
}

${ }^{1}$ A.I. Virtanen Institute for Molecular Sciences, University of Eastern Finland, Kuopio, Finland, ${ }^{2}$ Center of Diagnostic Imaging, Department of Cyclotron and Radiopharmacy, Kuopio University Hospital, Kuopio, Finland

Sustained inflammation in the injured cortex is a promising therapeutic target for disease-modification after traumatic brain injury (TBI). However, its extent and dynamics of expansion are incompletely understood which challenges the timing and placement of therapeutics to lesioned area. Our aim was to characterize the evolution of chronic inflammation during lesion expansion in lateral fluid-percussion injury (FPI) rat model with focus on the MRI-negative perilesional cortex. T2-weighted MR imaging (T2w MRI) and localized magnetic resonance spectroscopy (MRS) were performed at 1, 3, and 6 months post-injury. End-point histology, including Nissl for neuronal death, GFAP for astrogliosis, and Prussian Blue for iron were used to assess perilesional histopathology. An additional animal cohort was imaged with a positron emission tomography (PET) using translocator protein $18 \mathrm{kDa}$ (TSPO) radiotracer $\left[{ }^{18} \mathrm{~F}\right]$-FEPPA. T2w MRI assessed lesion growth and detected chronic inflammation along the lesion border while rest of the ipsilateral cortex was MRI-negative (MRI-). Instead, myo-inositol that is an inflammatory MRS marker for gliosis, glutathione for oxidative stress, and choline for membrane turnover were elevated throughout the 6-months follow-up in the MRI- perilesional cortex (all $p<0.05$ ). MRS markers revealed chronically sustained inflammation across the ipsilateral cortex but did not indicate the upcoming lesion expansion. Instead, the rostral expansion of the cortical lesion was systematically preceded by a hyperintense band in T2w images months earlier. Histologic analysis of the hyperintensity indicated scattered astrocytes, incomplete glial scar, and intracellularly packed and free iron. Yet, the band was negative in $\left[{ }^{18} \mathrm{~F}\right]$-FEPPA-PET. $\left[{ }^{18} \mathrm{~F}\right]$-FEPPA also showed no cortical TSPO expression within the MRS voxel in MRI- perilesional cortex or anywhere along glial scar when assessed at 2 months post-injury. However, $\left[{ }^{18} \mathrm{~F}\right]$-FEPPA showed a robust signal increase, indicating reactive microgliosis in the ipsilateral thalamus at 2 months post-TBI. We present evidence that MRS reveals chronic posttraumatic inflammation in MRI-negative perilesional cortex. The mismatch in MRS, MRI, and PET measures may allow non-invasive endophenotyping of beneficial and detrimental inflammatory processes to aid targeting and timing of anti-inflammatory therapeutics. 


\section{INTRODUCTION}

The global incidence of traumatic brain injury (TBI) is estimated to be 69 million each year. In the United States 1.7-2.5 million people sustain a TBI annually and over 5.3 million are living with TBI-related disability [2005-2010 statistics; Centers for Disease Control and Prevention (CDC, 2017)]. The disability may emerge years after the injury, and apparently derives from slow detrimental pathologic processes and accumulated load of stressors - such as neuroinflammation - that could potentially be seized or alleviated by interventions, even after the acute post-injury phase (Pearn et al., 2017; Sun et al., 2017; Missault et al., 2018).

Lateral fluid-percussion injury (FPI) rat model for TBI is a clinically relevant well-characterized animal model of closedhead injury in humans (Thompson et al., 2005). The primary brain contusion site atrophies within the first weeks to months after the injury, and gradually forms a cerebrospinal fluid (CSF) -filled cavity upon debris clearance (Pierce et al., 1998; Immonen et al., 2009a). At chronic stage the "perilesional" or "pericontusional" cortex beyond the lesion edge and beyond the glial scar may appear normal in conventional T2w structural MRI. However, histologic analyses have shown widespread perilesional neuroinflammation (Chen et al., 2003; NdodeEkane et al., 2018), and fMRI has demonstrated functional defect (Niskanen et al., 2013). Moreover, perilesional cortex undergoes cellular and vascular degenerative as well as plastic repair processes which progress in parallel with local innate inflammatory response for months after injury (Hayward et al., 2011; Jullienne et al., 2016; Kyyriainen et al., 2017; van Vliet et al., 2017; Ndode-Ekane et al., 2018; Pitkanen et al., 2018).

Inflammation is postulated to play a crucial role in the long-term outcome of TBI, contributing to the development of posttraumatic epilepsy and increasing the risk of cognitive deficits and other morbidities (Burda et al., 2015; Corps et al., 2015; Hinson et al., 2015; Lucke-Wold et al., 2015; Ali et al., 2018; Kokiko-Cochran and Godbout, 2018; Missault et al., 2018). Various in vivo imaging approaches have been applied to assess the posttraumatic neuroinflammatory state. MRI studies utilizing intravascular contrast agents have detected chronically elevated blood-brain-barrier (BBB) permeability in the perilesional cortex ( $\mathrm{Li}$ et al., 2016; Yoo et al., 2018) suggested to be associate with local inflammatory response (Dadas and Janigro, 2018). A proton MRS indicated an elevation in glial marker myo-inositol in the ipsilateral cortex acutely and during the first 2 weeks after controlled cortical impact injury (CCI) (Xu et al., 2011; Harris et al., 2012). PET and autoradiography studies utilizing translocator protein $18 \mathrm{kDa}$ (TSPO) radiotracers specific for reactive microglia and astroglia have demonstrated massive inflammation due to phagocytic microglia, peaking within the lesion on post-injury days 4-6. This is followed by a decaying signal from reactive astrogliosis at lesion edges, or microgliosis along injured axons, during the following weeks (Yu et al., 2010; Wang et al., 2014; Israel et al., 2016). However, the understanding of the evolution of chronic posttraumatic perilesional inflammation is far from complete.
We hypothesized that MRS profile of myo-inositol (Ins), glutathione (GSH), and total choline (GPC+PCho) will detect inflammation in MRI-negative cortex and that the increase or decrease of the concentration of the markers over time will differentiate whether the inflammation is sustained or attenuated. We refer to the combined information of glia marker Ins, oxidative stress marker GSH and membrane turnover marker GPC-PCho as inflammatory MRS profile. We further hypothesized that the inflammatory MRS profile will associate with the lesion expansion rate and the glial scar properties which are influenced by early and late immune responses (Sofroniew and Vinters, 2010). We assessed (a) lesion growth rate, (b) ${ }^{1} \mathrm{H}$ MRS neurochemical profile of the perilesional cortex at 1,3 , and 6 months after lateral FPI, (c) radiological inflammation characteristics around the lesion by T2w structural MRI, and (d) validated the imaging findings with the end-point histopathology. To complement the MRS and MRI findings, we collected PET data obtained with TSPO radiotracer $\left[{ }^{18} \mathrm{~F}\right]$-FEPPA in another animal cohort, and describe the chronic cortical TSPO expression along with structural MRI characterization.

\section{MATERIALS AND METHODS}

\section{Animals}

Adult male Sprague Dawley rats (Harlan Netherlands B.V., Horst, Netherlands, $n=43$, weight $387 \pm 23 \mathrm{~g}$ at time of TBI) were used for the study. Rats were housed in individual cages under controlled conditions (temperature $22 \pm 1{ }^{\circ} \mathrm{C}$, humidity 50$60 \%, 12 \mathrm{~h}$ light/12 h dark cycle) with ad libitum access to food pellets and water. All animal procedures were approved by the Animal Care and use Committee of the University of Eastern Finland and done in accordance with the guidelines of European Community Council Directives 2010/63/EU and 86/609/EEC. Animals were randomly grouped into TBI $(n=31)$ and shamoperated control $(n=8)$ groups. Four 8.5 months old naïve rats served as additional age matched non-operated control group for the 6 months post-operation data.

\section{Lateral Fluid-Percussion Injury}

Traumatic brain injury was induced by lateral fluid-percussion described previously (McIntosh et al., 1989; Kharatishvili et al., 2006). Briefly, rats were anesthetized by injecting intraperitoneally $(6 \mathrm{ml} / \mathrm{kg})$ a cocktail of sodium pentobarbital (58 mg/kg), magnesium sulfate $(127.2 \mathrm{mg} / \mathrm{kg})$, propylene glycol (42.8\%), and absolute ethanol (11.6\%). A craniectomy of $5 \mathrm{~mm}$ diameter was drilled between bregma and lambda with a trephine on the left convexity (anterior edge $2.0 \mathrm{~mm}$ posterior to the bregma; lateral edge adjacent to the left lateral ridge) leaving the dura intact. Injury impact was induced by transient pressure fluid pulse of $(21-23 \mathrm{~m} / \mathrm{s})$ by using fluid percussion device (AmScien Instruments, Richmond, VA, United States) against exposed dura. Impact pressure was adjusted to $3.5 \mathrm{~atm}$ in order to induce severe TBI. Pressure delivered ranged from 3.1 to $3.4 \mathrm{~atm}$. Sham-operated controls received identical surgical procedure without impact. 


\section{Magnetic Resonance Imaging (MRI)}

Magnetic resonance imaging measurements were performed on 9.4 T horizontal magnet with linear volume transmit/quadrature surface receiver coil system (Bruker, ParaVision 5.1 system, RAPID coils). MRI scans were acquired at 1,3 , and 6 months from the day of injury or sham-operation. Anesthesia was induced with isoflurane (1.5\%) in mixture of $70 \% \mathrm{~N}_{2} \mathrm{O}, 30 \%$ $\mathrm{O}_{2}$. Animals were secured to an animal holder with ear bars and bite bar. The holder was electronically heated to maintain a body temperature of $37^{\circ} \mathrm{C}$ throughout scanning. Respiratory rate was kept between 60 and 70 per min by adjusting isoflurane anesthesia level.

T2-weighted (T2w) coronal and horizontal multi-slice images were acquired with fast spin echo (TurboRARE) sequence with effective echo time $33 \mathrm{~m} / \mathrm{s}$, repetition time $2500 \mathrm{~m} / \mathrm{s}$, field of view $30 \times 30 \mathrm{~mm}^{2}$ covered with $256 \times 256$ data points, RARE factor 8 (echo train length 8 echoes, shortest echo time $10.7 \mathrm{~m} / \mathrm{s}$, and echo spacing $11 \mathrm{~m} / \mathrm{s}), 6$ averages and scan time $8 \mathrm{~min}$. Data set was composed of consecutive 17 coronal slices or 14 horizontal slices with thickness of $0.7 \mathrm{~mm}$ with no gaps. Cortical lesion volumes were obtained by intensity threshold based analysis of T2w images using in house written Matlab based analysis toolbox Aedes ${ }^{1}$. Two regions of interest (ROIs) were drawn in each animal: multi slice template of the ipsilateral cortex that mirrored the shape of the intact contralateral cortex, and a reference region in contralateral cortical gray matter. Pixels deviating $>4$ times the standard deviation (SD) from the contralateral cortical gray matter intensity were classified as abnormal tissue (i.e., the lesion). Lesion volume was then measured within the ROI template of intact, ipsilateral cortex. Subcortical atrophy was not included. Output is the "lost cortical volume." The reproducibility in volumetric measurement was assessed by repeated $(N=5)$ volume measurements in the same animal with large cavity type lesion. Two times the SD $\left(2 \mathrm{~mm}^{3} \times 0.75 \mathrm{~mm}^{3}\right)$ was then considered as an error margin for the volume measurement. Thus, the increase in volume $>1.5 \mathrm{~mm}^{3}$ was considered as reliably detected growth in lesion volume.

In the rats undergoing the PET examination, 3D T1wt anatomical images were acquired with fast imaging with steady state precession (FISP) sequence with TR $8 \mathrm{~m} / \mathrm{s}$, TE $4 \mathrm{~m} / \mathrm{s}$, flip $15^{\circ}, 175 \mu \mathrm{m}^{3}$ resolution by FOV $40 \mathrm{~mm} \times 40 \mathrm{~mm} \times 40 \mathrm{~mm}$ and $228 \times 228 \times 228$ matrix, $50 \mathrm{kHz}$ spectral width and 3 averages (the contrast is a function of T1 and T2*). FISP images were used as anatomical reference images for PET data.

\section{Magnetic Resonance Spectroscopy}

Localized (single voxel) spectroscopy was performed in the perilesional cortex using PRESS sequence with echo time $11 \mathrm{~m} / \mathrm{s}$, repetition time $2500 \mathrm{~m} / \mathrm{s}$, bandwidth $4 \mathrm{kHz}$ covered with 2048 points, and VAPOR (Tkac et al., 1999) water suppression. Fieldmap based shimming (MAPSHIM software package, Paravision 5.0, Bruker; Germany) was conducted to achieve water line width $<18 \mathrm{~Hz}$. MRS was acquired at 1,3 , and 6 months post-injury. Single voxel $(1 \mathrm{~mm} \times 3 \mathrm{~mm} \times 5 \mathrm{~mm})$

${ }^{1}$ http://aedes.uef.fi/ was placed in perilesional cortex avoiding CSF shown in Figure 4A, and 320 or 640 averages were collected. Reference water peak was acquired using the same pulse sequence without water supression. Figure 4B displays a representative spectrum, including the LCmodel (Provencher, 2001) fit and the residue. CSF volume fraction was quantified from $\mathrm{T} 2 \mathrm{w}$ images as an overlap of cystic lesion or ventricle with the MRS voxel. All metabolite concentrations are shown as absolute concentrations with CSF-volume fraction correction. Also, the fact that reference water peak is higher due to CSF fraction within the voxel (tissue water content $80 \%$ while CSF water content $99 \%$ ) is corrected for. As an alternative approach (results in Supplementary Data) concentrations were calculated relative to the total creatine $(\mathrm{Cr}+\mathrm{PCr}$; peak at 3.04). This is another way to account for the tissue atrophy, but it relies on the assumption that total creatine reflects the total cell number, and does not change as a disease effect. The spectra were analyzed with LC model and only results from metabolites with Cramér-Rao lower bound (SD\%) $<20$ were included in further analysis. SD\% were $10.5 \pm 3.0 \%$, $11.0 \pm 3.3 \%$, and $10.0 \pm 2.1 \%$ for Ins $(1,3$, and 6 months, respectively), $15.9 \pm 3.8 \%, 17.3 \pm 4.3 \%$, and $14.9 \pm 2.9 \%$ for GSH, and $10.3 \pm 1.7 \%, 9.5 \pm 1.9 \%$, and $9.2 \pm 1.8 \%$ for GPC+PCho, indicating that their concentrations could be reliably quantified with LCmodel fit.

\section{Positron Emission Tomography (PET) With $\left[{ }^{18} \mathrm{~F}\right]-\mathrm{FEPPA}$}

Small supplementary cohort of rats with lateral FPI $(n=3)$ was operated for $\left[{ }^{18} \mathrm{~F}\right]$-FEPPA-PET follow-up performed at 2 and 6 week $(n=1)$ or 4 and 8 week $(n=2)$ post-injury. Radiosynthesis of the TSPO radioligand $\left[{ }^{18} \mathrm{~F}\right]$-FEPPA was carried out as described by Wilson et al. (2008) with some modifications in the protocol.

Positron emission tomography scans were performed on Inveon DPET scanner (Siemens Medical Solutions, Knoxville, TN, United States). Anesthesia was induced with isoflurane $(1.5 \%)$ in mixture of $70 \% \mathrm{~N}_{2} \mathrm{O}, 30 \% \mathrm{O}_{2}$, rats were secured to an animal holder and the tail vein cannula was inserted. The holder was heated with warm water circulation to maintain a body temperature of $37^{\circ} \mathrm{C}$. Respiratory rate was monitored and maintained between $55-70$ by adjusting the anesthesia level. A 90 min dynamic PET was acquired after $28.8 \pm 2.2 \mathrm{MBq}$ $\left[{ }^{18} \mathrm{~F}\right]$-FEPPA tail-vein injection. Injection volume was $0.2-0.4 \mathrm{ml}$ and it was given as $30 \mathrm{~s}$ injection. Frames of the last $40 \mathrm{~min}$ were summed for the maps and activity quantification. After PET acquisition the animal holder with the rat was inserted to adjacent CT scanner (Flex X-O, Gamma Medica-Ideas, Northridge, CA, United States) and CT images were acquired for structural reference. Reconstruction for the PET data was done using OSEM-2D algorithm (16 subsets and 4 iterations) after Fourier rebinning. Dead time, decay, scatter and attenuation corrections were applied. Co-registration between the 3D MRI, CT and PET images were done manually using Carimas 2.9 (Turku PET Centre, Finland). CT to MRI co-registration allowed overlay of PET on MRI. MRI images were acquired in previous or following week in respect to the PET scan. PET activity 
maps are presented as percent of injected dose per cubic centimeter $(\% \mathrm{ID} / \mathrm{cc})$.

\section{Composite Neuroscore for Assessments of Somato-Motor Function}

Composite neuroscore tests were done to all animals at baseline (before TBI), and on day 2, day 7, and day 14 post-TBI to assess injury severity and motor recovery. Briefly, animals were scored from 0 (severely impaired) to 4 (normal). Animals were assessed for forelimb flexion (2 indices) during suspension by tail, hindlimb flexion ( 2 indices) by lifting hind limbs up and back by tail of animal whilst forelimbs remained on hard surfaces, ability of animal to resist a lateral propulsion (2 indices) toward the left and right and angle board. A composite neuroscore (0-28 points) was generated by combining the scores for each of seven tests (Nissinen et al., 2017). To investigate if the recovery rate of sensory-motor functions could predict the type of the developing lesion or the inflammatory response detected by MRS, we calculated a measure for the degree of recovery in NS. The motor recovery was calculated as a difference in neuroscore on day 14 and day 2 which was normalized to the magnitude of impairment on day 2, that is [NS(day 14)-NS(day 2)]/[NS(baseline)-NS(day 2)].

\section{Tissue Processing and Histology}

In the end of the 6-months follow-up, rats were perfused transcardially according to a paraformaldehyde fixation protocol described previously (Kharatishvili et al., 2007). Brains were removed and postfixed in 4\% paraformaldehyde (PFA) for $4 \mathrm{~h}$, cryoprotected in $20 \%$ glycerol in $0.02 \mathrm{M}$ potassium phosphate buffer (pH7.4) for $48 \mathrm{~h}$, frozen in dry ice and stored at $-70^{\circ} \mathrm{C}$. Brains were sectioned into coronal plane (1-in-10 series, $25 \mu \mathrm{m}$ thick) on sliding microtome. First series of brain sections was collected in $10 \%$ formalin at room temperature and remaining series were stored in tissue collection solution (30\% ethylene glycol, 25\% glycerol in $0.05 \mathrm{M}$ sodium phosphate buffer) at $-20^{\circ} \mathrm{C}$ until further processing.

One series of sections was stained for thionin (Nissl) to analyze the cytoarchitecture of the lesioned cortex. An adjacent series of selected sections was stained for Perl's Prussian blue to identify ferritic iron as described previously (Liu et al., 2014). Briefly, sections were incubated in freshly prepared solution of $5 \%$ potassium hexacyanoferrate trihydrate and 5\% hydrochloric acid for $30 \mathrm{~min}$ followed by rinsing with water and counterstaining with nuclear fast red. To assess the glial scar characteristics and putative reactive astrogliosis in the perilesional cortex, selected sections were stained for glial fibrillary acidic protein (GFAP). Free-floating sections were immunohistochemically stained using monoclonal mouse anti-mouse GFAP antibody (\#814369, Boehringer Mannheim, Germany). Briefly, the sections were treated with $1 \%$ hydrogen peroxide, washed in $0.02 \mathrm{M} \mathrm{KPBS}$, $\mathrm{pH} 7.4$, and blocked in 10\% normal horse serum (NHS) and $0.5 \%$ TritonX-100 in KPBS. Sections were then incubated for 2 days $\left(\right.$ at $\left.+4^{\circ} \mathrm{C}\right)$ in primary antibody (1:2000) in 1\% NHS and $0.5 \%$ TritonX-100 in KPBS. Thereafter, the sections were washed three times ( $2 \%$ NHS in KPBS), and incubated for
$1 \mathrm{~h}$ in biotinylated anti-mouse IgG (1:200, \#BA-2000, Vector Laboratories, Burlingame, CA, United States) diluted in 1\% NHS, $0.3 \%$ TritonX-100 in KPBS. Sections were washed again and incubated for $45 \mathrm{~min}$ in avidin-biotin-peroxidase complex according to manufacturer's instructions (Standard ABCkit, \#PK-4000, Vector Laboratories). Then the incubations in the secondary antibody and in the avidin-biotin solution were repeated. The peroxidase activity was visualized with $0.05 \%$ 3,3'-diaminobenzidine (DAB, Pierce Chemicals, Rockford, IL, United States) solution containing $0.04 \% \mathrm{H}_{2} \mathrm{O}_{2}$ and 0.02 $\mathrm{M}$ KPBS. The sections were mounted on gelatin-coated slides, dried overnight, and intensified with osmium tetroxide $\left(\mathrm{OsO}_{4}, \# 191970\right.$, Electron Microscopy Sciences, Hatfield, PA, United States) and thiocarbohydrazide (\#21900, Electron Microscopy Sciences, Hatfield, PA, United States) according to the method of Lewis et al. (1986). Finally, the slides were covered using DePex ${ }^{\circledR}$.

\section{Statistics}

Statistical analyses were performed using IBM SPSS Statistics (v.21). Differences in MRS neurochemical concentrations between TBI, sham and naïve groups were assessed with Kruskall-Wallis followed by a post hoc analysis using the Mann-Whitney $U$-test (applicable for non-normally distributed data). To assess the change over time in neurochemical concentrations between consecutive time points ( 1 vs. 3 months and 3 vs. 6 months) Wilcoxon's test was performed and corrected for multiple comparisons (Bonferroni). Correlations between lesion volume and subsequent lesion growth, between apnea duration and lesion volume, between subacute Ins concentration and lesion volume, and between final GSH and $\mathrm{GPC}+\mathrm{PCh}$ concentrations and preceding lesion growth were calculated using Pearson and corrected for multiple comparisons (Bonferroni). Data are reported as mean $\pm \mathrm{D}$. $P$-values $<0.05$ were considered significant.

\section{RESULTS}

Acute post-impact mortality was $36 \%(11 / 31)$ in the TBI group and $0 \%(0 / 8)$ in sham-operated control group. Post-impact apnea was $31 \pm 17 \mathrm{~s}$ with two rats with apnea time $>50 \mathrm{~s}$. Acute postimpact seizure-like behaviors (jerks) occurred in 48\% (15/31) animals and their mean duration was $30 \pm 9 \mathrm{~s}$.

\section{Evolution of Cortical Lesion in T2w Structural MRI}

We measured the cortical lesion volume in $\mathrm{T} 2 \mathrm{w}$ images encompassing all MRI positive (MRI+) cortical areas (Figure 1). Even in the hands of one experienced technician, the same impact force produced by the fluid-percussion device $(3.27 \pm 0.08 \mathrm{~atm})$ resulted in a variable severity of cortical lesion within the TBI cohort large enough for detection of heterogeneity. Heterogeneity in early lesion growth rate is reflected by the lesion volume reached by 1 month post-injury (Figures 1, 2A). We classified the animals into three groups based on the lesion size at 1 month post-injury (Figure 2A). Consequently, 40\% (8/20) 

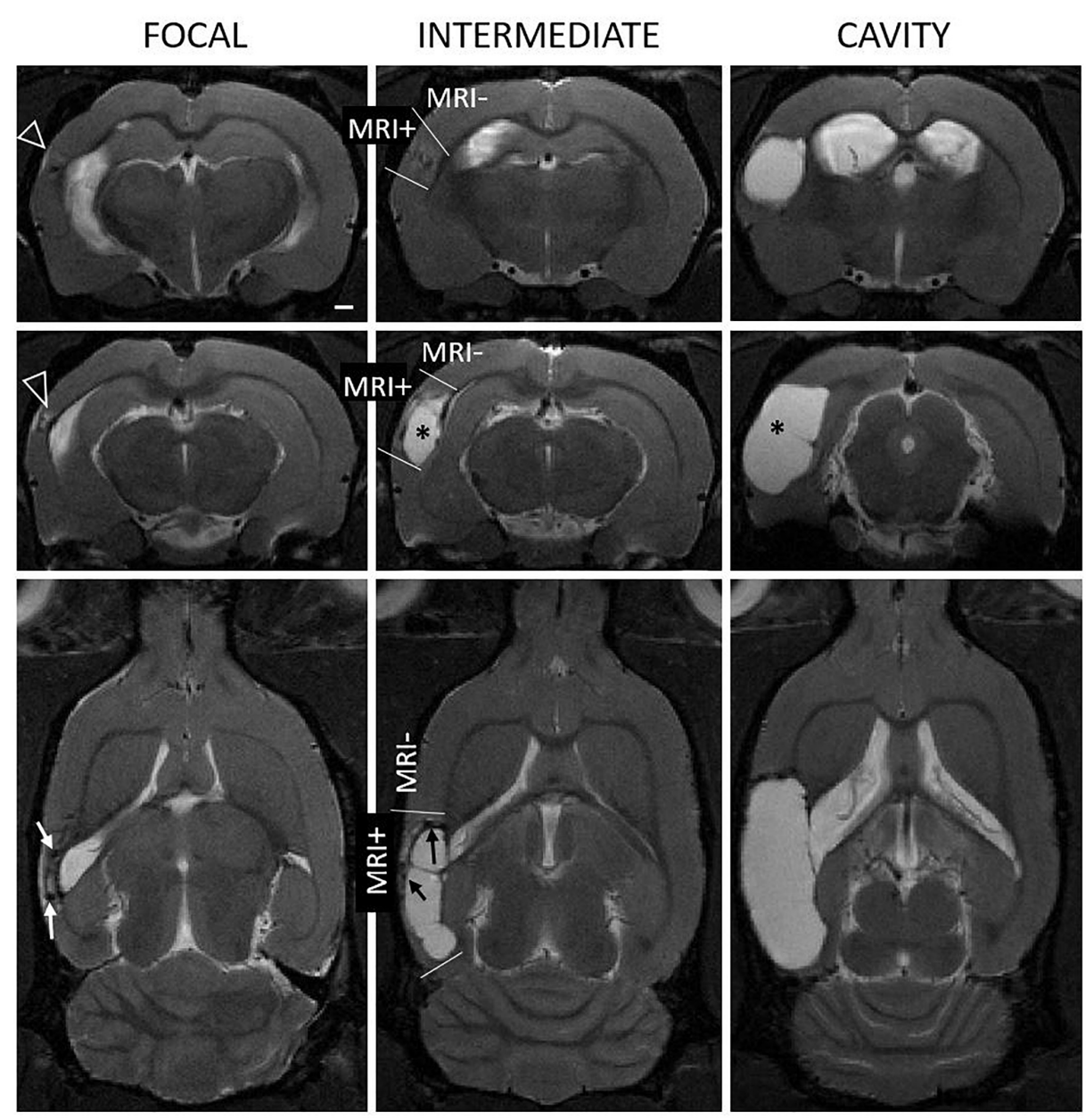

FIGURE 1 | Heterogeneity of the endophenotype of cortical lesion in T2-weighted images at 1 month after lateral fluid-percussion induced TBI. Panel shows representative examples of "focal" (left column), "intermediate" (mid column), and "cavity-forming" (right column) endophenotypes, highlighting the 3D extent of the cortical lesions (see also Figure 3). Top row: Coronal views at the level of the rostral lesion tip. Note the rostral T2-enhancement (white triangle). Middle row: Center of the cortical lesion. Bottom row: Horizontal view depicting the rostro-caudal lesion extent. Contusion site is atrophied and the cavity filled with cerebrospinal fluid (asterisk). In focal and intermediate cases, the lesion was still expanding at 1 month and showed prominent T2w hyperintensity in the surrounding cortex, suggesting on-going inflammation (open arrowheads). We also found hyperintensity in association with hypointense hematomas or iron deposits (black and white arrows). MRI negative (MRI-) cortex refers to areas with normal gray matter contrast while MRI positive (MRI+) cortex refers to all observable abnormalities. Scale bar equals $1 \mathrm{~mm}$.

of rats had developed a large $\left(>48 \mathrm{~mm}^{3}\right)$ CSF filled cystic lesion, extending throughout all cortical layers ("cavity"). $30 \%(6 / 20)$ of animals showed a small $\left(<20 \mathrm{~mm}^{3}\right)$ "focal" lesion. The remaining $30 \%(6 / 20)$ of rats had intermediate lesion volumes $\left(26-45 \mathrm{~mm}^{3}\right)$. Interestingly, the lesion had a heterogeneous, trabecular appearance, suggesting on-going necrosis and incomplete debris clearance. By 6 months, the "intermediate" cortical lesion endophenotype progressed to CSFfilled cavity. Figure 3 shows the lesion progression over time in representative cases of the "cavity," "intermediate," and "focal" endophenotypes. Multifocal hematomas in the contusion site were accompanied by hyperintense edema, and necrotic areas. This affected regions later atrophied into a lesion cavity. Narrow rim of cortical T2w-hyperintensity outside the primary lesion cavity indicative of local on-going inflammation was observable still 3 months post-injury. Rest of the ipsilateral cortex was MRI-negative (MRI-).

The subsequent growth of lesion cavity from 1 to 3 months, and thereafter, from 3 to 6 months post-injury varied greatly between the animals. The absolute volume of cortical tissue loss measured did not differ between groups. However, relative lesion volume loss between month 1 and month 3 was $6 \pm 6 \%$ in the cavity, $26 \pm 17 \%$ in the intermediate, and $115 \pm 137 \%$ in the focal group. Thereafter, from 3 to 6 months the relative increase of the lesion volume was $2 \pm 4 \%$ in the cavity, $1 \pm 2 \%$ in the intermediate, and $5 \pm 5 \%$ in the focal group. The lesion volume at 1 month did not correlate with the subsequent lesion growth (Figures 2A,B). The progression in lesion growth continued in $75 \%(15 / 20)$ of animals after 1 month. Of the 5 rats with no progression in lesion volume, 4 belonged into the cavity and 1 

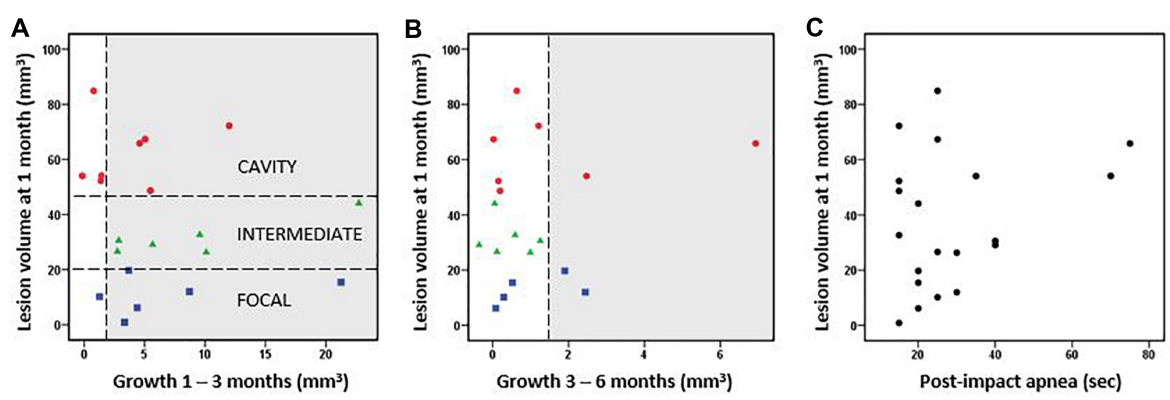

FIGURE 2 | Cortical lesion volume and growth. (A) Rats were classified into three groups based on the cortical lesion volume at 1 month post-injury [horizontal lines in A separate the cavity (red, $>48 \mathrm{~mm}^{3}$ lesion already at 1 month post-TBI), intermediate (green, 20-48 $\mathrm{mm}^{3}$ ), and focal (blue, $<20 \mathrm{~mm}^{3}$ ) groups]. The lesion volume at 1 month did not correlate with the subsequent lesion growth from 1 to 3 months post-injury (Pearson, $p>0.05$ ). (B) Lesion volume vs. lesion growth from 3 to 6 months post-injury. Thus, the growth of cortical lesion after the 1st post-injury month as calculated in cubic millimeters did not differ between the groups. Proportionally, however, the lesion volume two-folded in the focal group and increased almost $30 \%$ in the intermediate group between 1-6 months, whereas it stayed almost the same in the cavity groups. The growth $>1.5 \mathrm{~mm}^{3}$ was considered as a true positive finding and highlighted in gray. The possible growth $<1.5 \mathrm{~mm}^{3}$ is within the error margin of the quantification method. (C) Post-impact apnea duration did not correlate with the volume of cortical lesion (Pearson, $p>0.05$ ).

into the focal group. Between 3 and 6 months, the growth of lesion volume exceeded $1.5 \mathrm{~mm}^{3}$ (detection limit) only in $20 \%$ $(4 / 20)$ of animals.

Since prolonged post-impact apnea may present a "second hit" and aggravate the aftermath of primary impact, we examined its association with the lesion volume. Apnea duration did not correlate with the lesion volume at 1 month post-injury (Figure 2C) or with lesion growth. However, the two cases with the longest apnea duration (>60 s) did develop large lesion cavities, and they showed a continuous chronic growth of lesion volume between 3 to 6 months (Figures 2B,C). Magnitude of impact pressure did not associate with the developing lesion volume.

\section{MRS Inflammatory Profile Stays Elevated From 1 to 6 Months in the MRI Negative Cortex}

Magnetic resonance spectroscopy was performed to detect any subtle cortical inflammation. The MRS voxel was placed to MRI- perilesional cortex (voxel location depicted in Figure 4A). MRS revealed elevated concentrations of inflammation markers already at 1 month post-injury as compared to shamoperated experimental controls. Concentrations of glia marker Ins, antioxidant GSH and membrane turnover indicator GPC+PCho stayed elevated for 6 months after injury suggesting sustained inflammation (Figures 4B-E). Concentrations in shams 6 months post-operation did not differ from those in age matched naïve rats. Thus, unlike the T2w MRI, the spectroscopy could detect the chronic inflammation even at 6 months after injury.

\section{Association of the Dynamics of the Inflammatory MRS Profile to the Cortical Lesion Growth Rate}

Next, we investigated how the inflammatory MRS profile reflected the early lesion growth until 1 month and the subsequent chronic lesion growth between 1-6 months. Findings are summarized in Figures 5A-C. Volume of the cortical lesion at 1 month associated with the 1-month Ins levels, suggesting that the early growth rate relates to the amount of glia in MRI- cortex. The MRS markers analyzed at 1 month postinjury did not predict the subsequent growth of cortical lesion. However, the levels of MRS markers at the chronic 6-month time point associated with the magnitude of the preceding lesion growth. GSH and GPC+PCho concentrations were the highest in animals with the greatest lesion growth during the preceding 1 to 6 months.

We found a remarkable variation in the dynamics of MRS markers in individual animals during the follow-up. Particularly, the two extremities were conspicuous: animals with the Ins, GSH and GPC+PCho concentrations progressively increasing or remaining at a high level, and animals with the levels of MRS markers decreasing over time. Therefore, we assessed whether the specific patterns in MRS markers would associate with the structural endophenotype of the cortical lesion and the lesion growth rate. Figures 5D-F shows the dynamics of different MRS inflammatory markers in different structural endophenotypes. In the cavity forming cases the levels of perilesional Ins between 13 months post-TBI remained rather stable. In the animals with focal lesions the direction of change in absolute Ins levels varied (Figure 5D). When the level of Ins were normalized to total creatine (Supplementary Figure 1), they decreased over time in the cavity group, indicating attenuating of the inflammation (Supplementary Figure 1A). Antioxidant GSH was the highest in the cavity forming cases, but its concentration or dynamics did not differ from that of the focal endophenotype. Total choline, GPC+PCho, a marker of membrane turnover, showed a late increase, starting at 3 months, and remained elevated at 6 months. It did not show endophenotype-specific dynamics. Thus, the dynamics of individual compounds Ins, GSH or GPC+PCho could not be linked with the structural endophenotype.

Unsupervised hierarchical clustering was performed to test if the three perilesional MRS markers as a combination would cluster the three endophenotypes of cortical pathology correctly within the TBI group. While the sham-operated experimental 

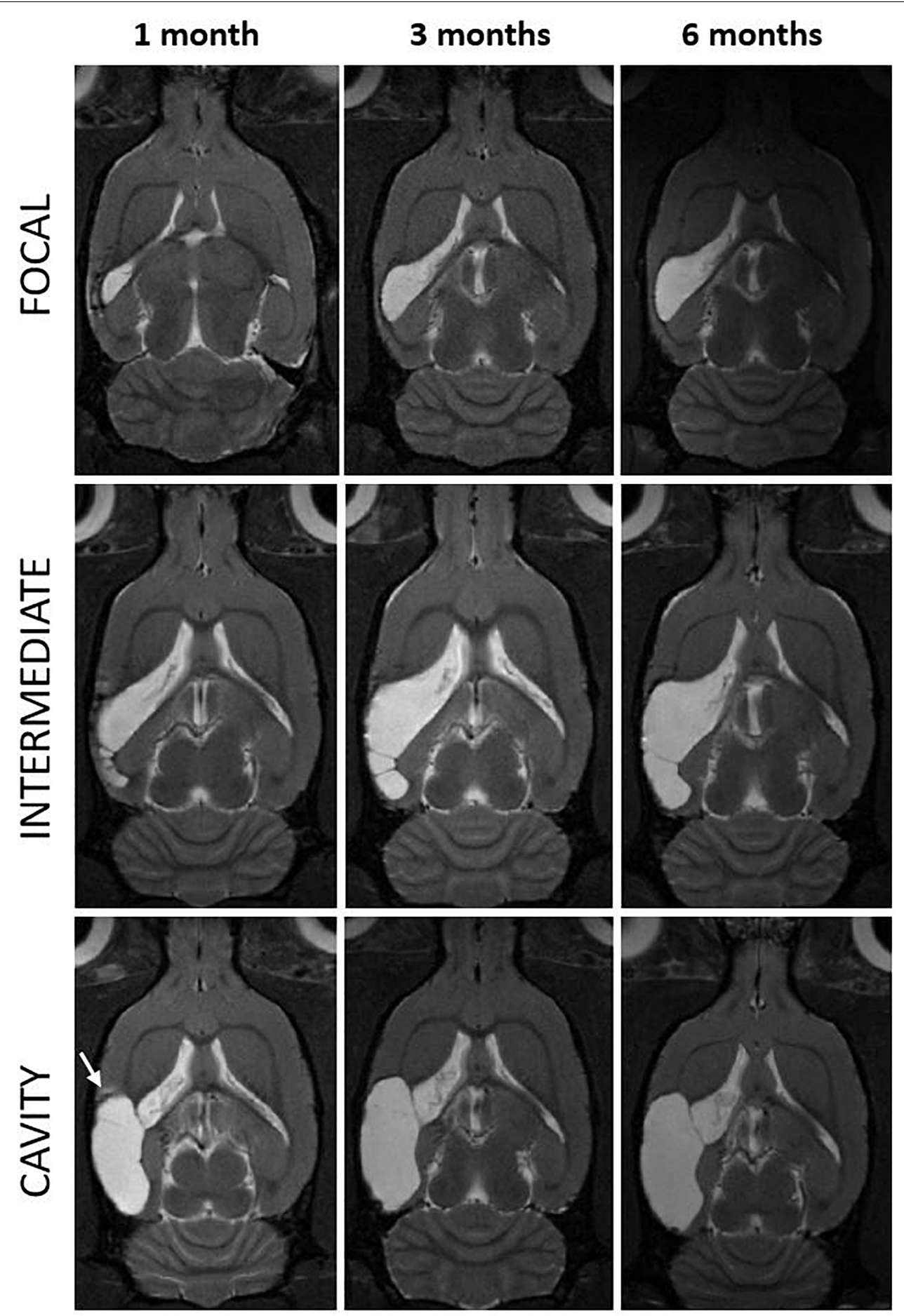

FIGURE 3 | Progression of the cortical lesion from 1 to 6 months post-injury in focal, intermediate and cavity endophenotypes. T2-weighted horizontal images of three representative rats with lateral FPI (rows) over the 6-months follow-up (columns) are showing different rates of expansion of the cortical lesion. Lesion in a cavity case was already close to its maximal size by 1 month (left and bottom) while the focal and intermediate cases display slower rate of tissue atrophy and show trabecular lesions. They also show multisite hematomas and edema indicating on-going inflammation at 1 month. Note that also in the cavity case, at the rostral aspect of the cortical lesion there is MRI-detectable T2w hyperintensity at 1 month post-TBI (arrow). The MRI signs of inflammation are abolished by 3-6 months. Lesion has expanded over the areas that were abnormal in MRI at 1 month post-injury. In (bottom right) the cortical lesion cavity now covers also the area of the rostral T2w hyperintensity present at 1 month. Even though the rostral lesion growth in most of the cases was below the detection limit of the volumetric analysis $\left(<1.5 \mathrm{~mm}^{3}\right)$, it was apparent in visual analysis. 

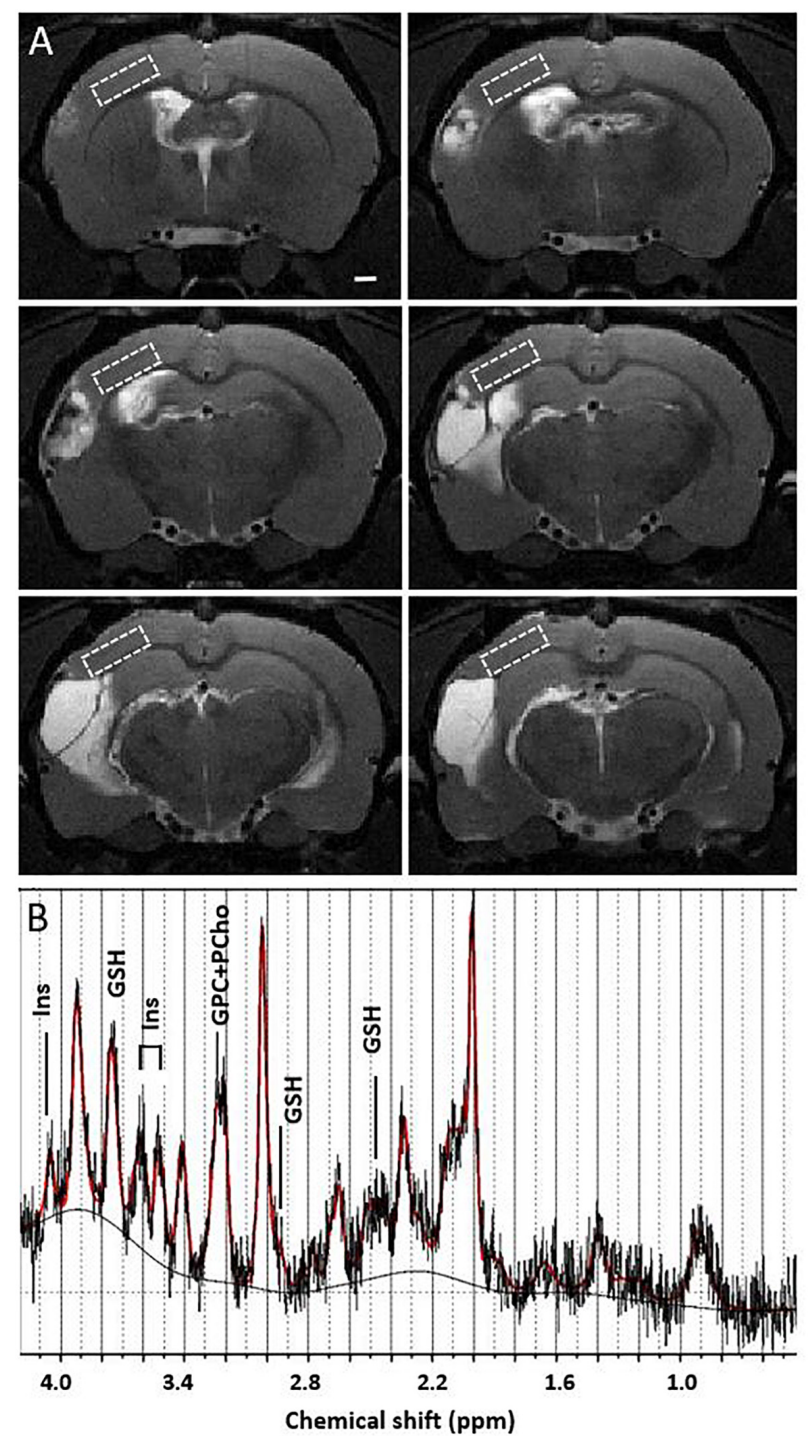

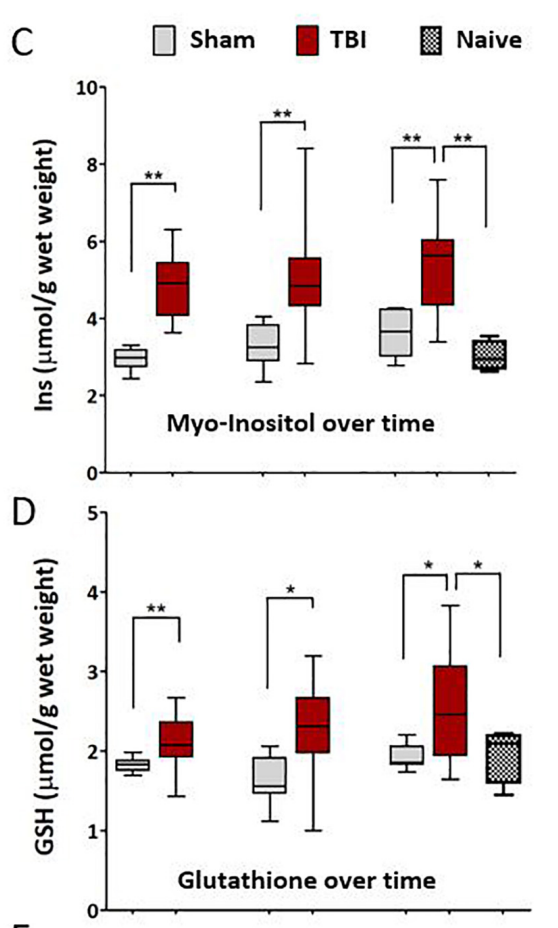

E

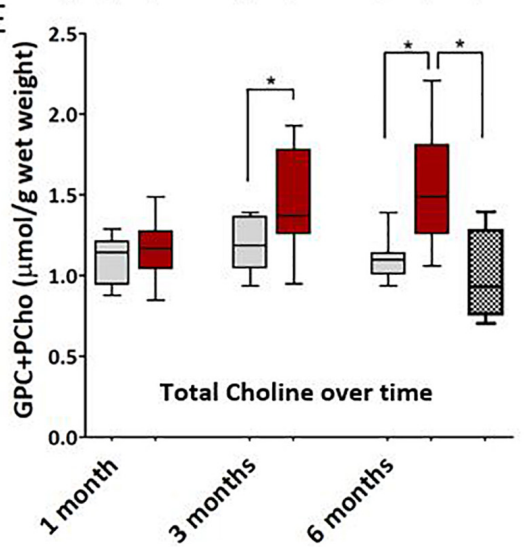

FIGURE 4 | Magnetic resonance spectroscopy detects chronic cortical inflammation in MRI-negative perilesional cortex. (A) Location of the MRS voxel (white dashed square) at different rostro-caudal levels in the dorsal perilesional cortex in a representative animal at 6 months after TBI. Voxel was placed over the MRItissue in the T2w images. Note that at this chronic follow-up point the cortical thinning and progressive atrophy restricted the voxel size which had to be anticipated in the planning of the study. The strategy for voxel placement was to exclude the perilesional glial envelope and keep the distance from the lesion edge constant. In animals with the most extensive lesions and heavily enlarged ventricles, the overlap of the voxel with subcortical white matter (external capsule) and CSF-filled cavity could not be completely avoided, which was accounted for in the analysis. (B) Representative data showing a raw spectrum (black), LCmodel fit (red), and fitted baseline (solid black). The inflammatory markers myo-inositol (Ins, glial marker), glutathione (GSH, astrocyte-produced antioxidant) and glycerophosphocholine+phosphocholine (GPC+PCho, a marker of membrane turnover, both proliferation and degradation) are assigned to the corresponding peaks. LCmodel fit succeeded with Cramér-Rao lower bound SD\% 10.5 \pm 2.8 for Ins, $16.1 \pm 3.8$ for GSH, and 9.6 \pm 1.8 for GPC+PCho. Absolute concentrations were corrected for the CSF volume fraction. Water reference is corrected for the $99 \%$ water content of CSF fraction while tissue water content was assumed to be 80\%. (C-E) Box-plots showing the perilesional concentrations of Ins, GSH and GPC+PCho at 1, 3, and 6 months post-injury. The indicators for gliosis and oxidative stress were elevated through the 6 months follow-up. However, the increase in choline was not apparent untill at 1 month post-TBI, suggesting a more delayed tissue plasticity after the initial gliosis. Number of animals was 20 in the TBI, 8 in the sham-operated control, and 4 in the naïve group. Difference between the sham and TBI groups was assessed with Kruskall-Wallis with Mann-Whitney post hoc analysis; statistical significances: ${ }^{*} p<0.05$ and ${ }^{* *} p<0.01$. No differences between the time points were found (Wilcoxon). Significances were corrected for multiple comparisons. The significances remained the same when calculated with the ANCOVA, including white matter volume fraction as covariate.

controls clustered separate from the animals with TBI based on the levels of Ins, GSH and GPC+PCho, the clustering did not separate the TBI endophenotypes correctly. Supplementary
Figures 2A,B shows the $3 \mathrm{D}$ clustering and the best of the dendrograms at 1,3 , and 6 months post-TBI. Taken together, the inflammatory MRS profile (Ins, GSH and GPC+PCho 

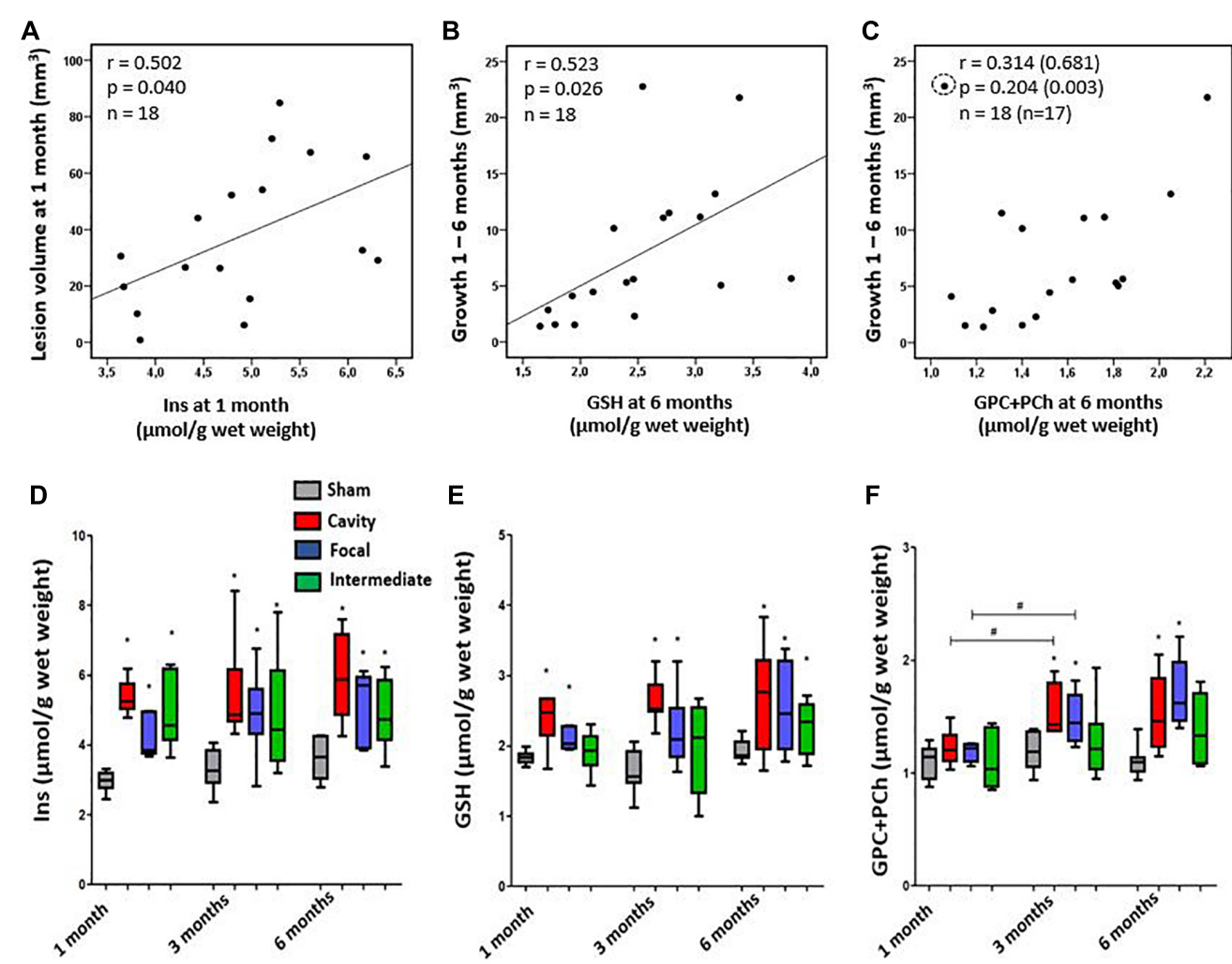

FIGURE 5 | (A-C) Association of cortical inflammatory MRS profile with the structural endophenotype and lesion growth. (A) Myo-Inositol (Ins) concentration correlated with the lesion size at 1 month post-injury, but not at later time points. (B) Glutathione (GSH) concentration at 6 months post-injury correlated with the preceding growth of cortical lesion from 1 to 6 months. Similarly, (C) the total choline (GPC+PCho) concentration at 6 months was higher in animals with more pronounced preceding lesion growth, but correlation was not significant unless an outlier (circled) was excluded (values in brackets). Pearson correlations (A-C) are no longer statistically significant after Bonferroni correction for multiple comparisons $(p>0.05)$. (D-F) Dynamics of the inflammatory MRS profile over time in each structural endophenotype. (D) Endophenotypes did not significantly differ in their Ins dynamics, although the group with focal lesion had the lowest Ins level at 1 month and showed a trend toward increased Ins levels thereafter. (E) Antioxidant glutathione (GSH) produced by astroglia was elevated in all endophenotypes, but it was the highest in the cavity forming group. Temporal dynamics of the MRS markers did not differ between the groups. (F) The level of total choline (GPC+PCho) did not differ from controls at 1 month post-TBI, but became elevated thereafter, indicating comparable membrane turnover in both the cavity forming and focal lesion groups. Statistical significances: ${ }^{*} p<0.05$ and (Mann-Whitney $U$-test between the groups); $\# p<0.05$ (Wilcoxon test, comparison of 1 and 3 months data from the same rats).

together) did not associate with the growth rate of the cortical lesion.

\section{Somatomotor Impairment and Spontaneous Recovery From Day 2 to Day 14 Post-TBI Did Not Predict the Endophenotype of Cortical Pathology at 1 Month}

Our data suggested that the rate of the progression in cortical lesion volume reports on the capacity of brain immunoresponse in a given rat. We then assessed whether progression of post-TBI somato-motor function (i.e., spontaneous recovery) would be linked to the evolution of the endophenotype of cortical lesion. Recovery of postTBI performance in neuroscore did not differ between the structural endophenotypes (Supplementary Figure 2C), neither did the severity of drop in the neuroscore from baseline to 2 day post-injury. Neuroscore results did not correlate with the developing lesion size or growth. Thus, neuroscore did not predict the lesion extent, its progression, or endophenotype. Neither did the neuroscore recovery index show any correlations with MRS inflammation markers. The impact pressure in FPI induction did not correlate with neuroscore results.

\section{Association of the Glia Scar Properties With the Lesion Expansion and With the Level of MRS Inflammatory Markers in MRI- Cortex}

Next, we studied the astroglia scar outlining and encapsulating the lesion. We hypothesized that the glia scar properties would differ in animals with different rate of lesion expansion. We also hypothesized that animals with leaky or incomplete glia scar would have higher levels of MRS inflammatory markers in MRI- parenchyma. 


\section{Glia Scar Histopathology at 7 Months Post-injury Showed Non-mature and Diffuse Glia Barrier at Site of On-Going Lesion Expansion During Preceding 3 to 6 Months}

Properties of the glia envelope were examined in the endpoint histology. A dense, tight and thin glial layer was assigned as a "mature" glial scar. A dispersed and diffuse gliosis was considered as a "maturing or immature" glial scar. Figure 6 shows the association between the Nissl cytoarchitecture at the lesion edge, GFAP characteristics and the structural MRI endophenotypes. Based on the GFAP and Nissl stainings all rats in the cavity group had a mature, narrow glial scar outlining all $3 \mathrm{D}$ aspects of the cortical lesion. The only exception was the rostral tip of the lesion, in which the scar remained diffuse. The animals in the focal group showed a thick cloud of GFAP+glia surrounding the lesion as well as the accompanying iron residues. Thus, even though the perilesional glial barrier was present in the focal group, it appeared diffuse and differed from the tightly packed mature envelope in the cavity group. All focal cases had dispersed gliosis rostral to the lesion.

Iron staining (Perl's Prussian Blue) showed both diffuse free iron and phagocyted intracellular iron at the areas of dispersed gliosis. Iron and iron aggregates were found embedded into the tightly packed perilesional mature glial scar as well as in gliaencapsulated isolated pockets. Histologically detected iron was also detected by the T2w MRI (Figure 6).

\section{T2-Hyperintense Band in MRI Next to the Glia Scar Preceded the Subsequent Lesion Growth}

We observed T2-hyperintensity anterior to the glial scar envelope that surrounded the rostral aspect of the lesion cavity in $75 \%$ of the TBI animals at 1-3 months post-injury. By 6 months
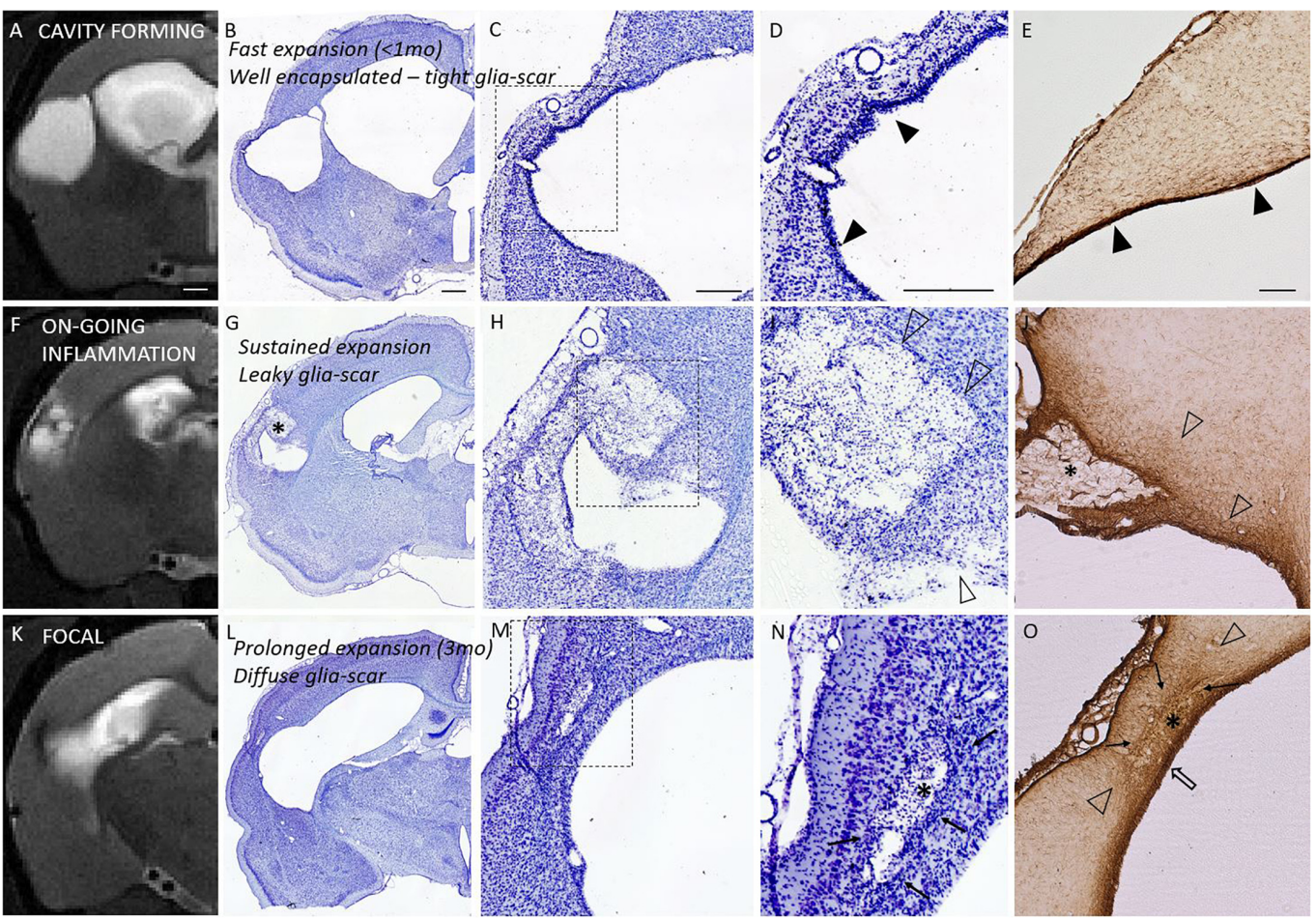

FIGURE 6 | Glia envelope maturation stages and their association with the structural endophenotypes. Panel shows three example animals representative of mature (top row), leaky (middle row), and diffuse (bottom row) glia scar around the lesion. T2-weighted MRI images (first column) are shown in parallel with the corresponding Nissl stained sections (2nd column and magnified views in 3rd and 4th column) as well as GFAP stained sections (last column). Top row shows tight narrow glia scar (solid arrowheads in panels D,E) completely encapsulating the lesion. This mature glia envelope is observed particularly in cavity forming endophenotype, in which cavity develops fast (within first weeks after injury, <1 month). Middle row shows a case, where glia scar is incomplete and leaky with dispersed gliosis (open arrowheads in panels $\mathbf{I}, \mathbf{J}$ ), there is on-going inflammation, and the lesion is showing sustained expansion in MRI. Bottom row shows focal endophenotype with lesion and all its' pockets surrounded by gliosis. While the glia encapsulates the lesions, the scar is not tight and narrow but a thick diffuse one, putatively still maturating (cloud of glia is indicated by solid arrows; thicker glia envelope indicated by open arrow). Iron residues are found at lesion edges in all

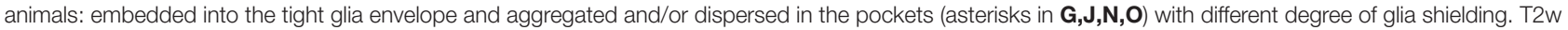
images are taken 6 months post-injury and the tissue is harvested 7 months post-injury. While on-going inflammatory T2w-MRI signature (F) is rare 6 months post-injury, it is observed in $75 \%$ of the TBI animals 1 month after injury. It manifests in the rostral tip of the lesion in cavity endophenotype, and more widely around the lesion in intermediate and focal endophenotypes. Nissl staining of the cytoarchitecture of the cortical lesion is shown with two magnifications (D,I,N are magnifications of the dashed square in panels $\mathbf{C}, \mathbf{H}, \mathbf{M}$, respectively). Scale bars equal $1 \mathrm{~mm}(\mathbf{M R l} ; \mathbf{A}, \mathbf{F}, \mathbf{K}), 1 \mathrm{~mm}(\mathrm{Niss}$ of whole hemisphere; $\mathbf{B}, \mathbf{G}, \mathbf{L}), 500 \mu \mathrm{m}$ (C,H,M), $500 \mu \mathrm{m}$ (D,I,N), and $200 \mu \mathrm{m}$ (GFAP; E,J,O). 
post-injury, the lesion cavity (i.e., tissue loss) had expanded to cover the entire area of T2 hyperintensity. All lesions with a rostral T2 hyperintensity expanded. Only a single focal case lacked the rostral T2 hyperintensity despite of rostral lesion expansion. Hyperintensity manifested always in the dorso-rostral aspect of the lesion located in the S1 barrel field cortex. The rostral edge of the lesion also showed remarkable iron accumulation visualized in T2w MRI. Figure 7 shows the MRI characteristics of the rostral perilesional cortex and corresponding Nissl, GFAP and Prussian blue stained sections.

\section{In vivo $\left[{ }^{18} \mathrm{~F}\right]$-FEPPA-PET Showed Perilesional Cortex to Be Void of TSPO Overexpression 6 Weeks Post-injury and Thereafter, Suggesting Negligible Levels of Pro-inflammatory Subtype of Reactive Microglia}

A small cohort of rats with lateral FPI was produced to probe the presence of reactive microglia and astroglia in the perilesional cortex over time by using in vivo $\left[{ }^{18} \mathrm{~F}\right]$-FEPPA-PET to map the binding of TSPO radiotracer. TSPO is overexpressed in the mitochondrial membrane of reactive M1 type microglia and reactive astroglia, but not in stable astroglial scar or other types of microglia, including anti-inflammatory M2 type microglia (Beckers et al., 2018). Perilesional cortex showed $\left[{ }^{18} \mathrm{~F}\right]$ FEPPA binding only at early 2-4 wk post-injury time points (Supplementary Figure 3). The cortical signal disappeared by 6 wk post-injury, and remained absent thereafter (Figure 8 and Supplementary Figure 3). In the same images we found massive TSPO signal in the ipsilateral thalamus, reporting on microgliosis (Figure 8 and Supplementary Figure 3). Interestingly, the MRS detected elevated astrocytic marker (Ins) and signs of oxidative stress $(\mathrm{GSH})$ in the $\left[{ }^{18} \mathrm{~F}\right]$-FEPPA-PET negative perilesional cortex. Moreover, the glia scar was devoid of any hot spots of TSPO expression despite the lesion growth was still continuing as verified by MRI follow up.

\section{DISCUSSION}

This study utilized three imaging approaches to reveal chronically on-going cortical posttraumatic inflammation in MRI- perilesional cortex and along the glia scar. Firstly, MRS detected elevated concentrations of inflammation indicating neurochemicals Ins, GSH and GPC+PCh in the MRI- perilesional cortex and, indeed, the pathological levels were sustained 1, 3, and 6 months after head injury. Secondly, T2w MRI a month after injury highlighted on-going inflammation next to the lesion border as hyperintensity which was abolished by 3 months and which preceded, and matched the area of the subsequent lesion growth. Thirdly, in vivo $\left[{ }^{18} \mathrm{~F}\right]$-FEPPA-PET showed negligible TSPO binding in the perilesional cortex or along the glia scar 1.5 months post-injury and thereafter suggesting absence of those types of reactive astro- and microglia that overexpress TSPO on their mitochondrial membrane. MRI follow-up also provided a comprehensive view to the progressive atrophy and yielded structural endophenotype classifications. The lesion expansion rate was found to be associated with the maturation stage of the glia scar evaluated by Nissl and GFAP staining. The lesion expansion rate associated only weakly with the dynamics of Ins, GSH and GPC+PCh in the preserved cortex.

\section{Spectroscopic Inflammation Markers Were Elevated Throughout the 6 Months Follow-Up in the MRI Negative Perilesional Cortex}

Spectroscopy voxel of $1 \mathrm{~mm} \times 3 \mathrm{~mm} \times 5 \mathrm{~mm}$ contained S1BF, dysgranular zone (S1DZ), front limb region (S1FL) (minor portion of hindlimb region S1HL), ending caudally in parietal association (PtA) and secondary visual (V2L) cortical regions. Lesion covered large portions of the primary and secondary somatosensory cortices ( $\mathrm{S} 1$ and S2), primary auditory cortex (Au1), dorsal and ventral areas of the secondary auditory cortex (AuD and $\mathrm{AuV})$, dorsal and rostral areas of posterior parietal cortex (PtPR and PtPR) extending often to ectorhinal cortex (Ect), perirhinal cortex (PRh), and entorhinal cortex (Ent) according to Paxinos rat atlas (Paxinos and Watson, 1998). Spectroscopy voxel avoided the lesion and probed the MRI- cortex revealing chronically elevated concentrations of inflammatory markers Ins, GSH and GPC+PCho. Thus, MRS revealed sustained chronic inflammatory-stage undetected by structural MRI.

\section{Methodological Considerations - MRS of Atrophied Cortex}

The target of the MRS measurement was the healthy appearing perilesional cortex, which has been largely overlooked in previous MRS studies. Lescot et al. (2010) focused their localized MRS study over the primary contusion in the cortex and excluded subcortical structures, but described only the first 7 days after lateral FPI. Harris et al. (2012) comprehensively described the changes in the entire neurochemical profile 2 weeks after the TBI in CCI rat model with localized $1 \mathrm{H}-\mathrm{MRS}$, but the horizontally localized voxel missed a major portion of the cortical areas we are covering, and included both the primary lesion and the subcortical structures that we are avoiding.

While assessing the neurochemical changes over time in TBI animals with progressive atrophy, it is crucial that the contributions of CSF volume fraction, and white matter into the cortical MRS voxel are accounted for in the analysis. We report here the absolute concentrations obtained from LCmodel analysis corrected for the CSF volume fraction and for the 99\% water content of CSF with tissue water content approximated to $80 \%$. Additional spectra obtained from a voxel located entirely within the lesion cavity showed that no Ins, GSH or choline signal arose from the CSF-filled-cavity (data not shown). In Supplementary Material we report the concentrations relative to total creatine, which in principle normalizes the results to the total cell count, and thereby takes into account the partial atrophy. Creatine levels, however, are susceptible for changes due to the pathology. Taken together, both the absolute 


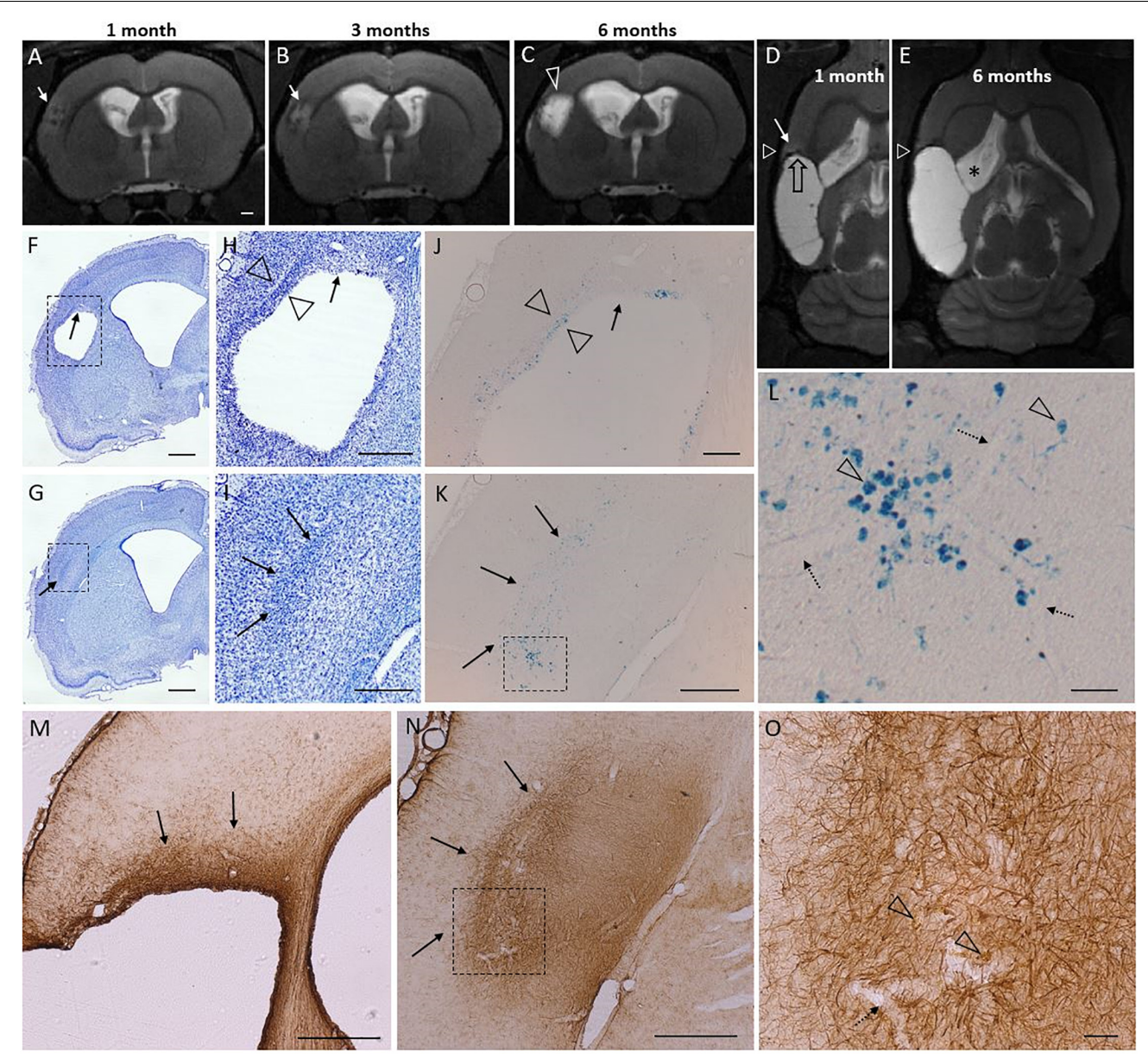

FIGURE 7 | T2 hyperintensity predicts the rostral lesion growth. Lesion continues to expand at its rostral tip which is characterized by glia cloud rather than a tight scar, and both diffuse and phagocyted iron. Iron is partly embedded into the glia scar. Subtle T2 hyperintensity is found to precede the lesion propagation and identify the rostral area to be atrophied in following months. Panel shows atrophy progression in T2-weighted (T2w) MRI in a representative rat from 1 to 6 months post-injury, and histological findings around the rostral tip of the lesion (7 months post-injury) in the same rat. [Two levels of Nissl (F-I), Perl's Prussian Blue (J-L), and

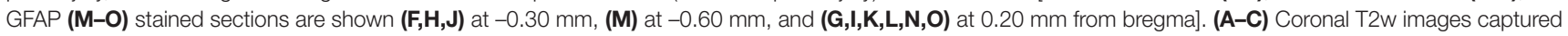
at the same rostrocaudal level of the same rat at 1, 3, and 6 months post-injury. (D,E) Horizontal images from the same rat, allowing elimination of any partial volume effects as a source of the hyperintensity. White triangle indicates the level of coronal images in panels (A-C). Black stripe (T2 hypointensity) along the lesion border was caused by iron residues (black open arrow). Cortical hyperintensity (white arrow in panel D) outside the rostral edge of the CSF-filled lesion predicted lesion progression over the following months [compare panels (D,E), rostral growth direction depicted by open black arrow in panel $\mathbf{D}$ ]. Expansion of the cortical lesion was accompanied by the continuous enlargement of the ipsilateral ventricle (asterisk). The hyperintense band was observed in $75 \%$ of the TBI animals at 1 month post-injury. By 6 months the hyperintense area had become merged into the lesion cavity. (F-I) Nissl and (M-O) GFAP stained sections of the same animal show a cloud of astrogliosis, incomplete glial envelope toward the dorso-rostral end of the lesion [arrows in panels (F,H,M)], and a thick glial scar next to it [open arrowheads in panel $\mathbf{( H )}$ ]. (G-I) Typical to rostral aspect of the lesion was a diffuse glial cloud, extending beyond the immediate lesion edge [arrows in panels (G-I,M,N)]. (J-K) Prussian blue staining shows the distribution of iron in corresponding locations. (L) A higher-magnification prohotomicrograph [dashed box in panel (K)] highlights phagocytosed iron (arrowheads), free diffuse iron, and iron associated with blood vessels (dotted arrows). (O) Open arrowheads point to iron within the rostral astroglial scar [dashed box in panel (N)]. In general, the glia envelope in all cavity cases was tight and narrow in all 3D-directions except rostrally. In the "tightly sealed" directions the lesion expansion seized by 1-3 months. The "leaky rostral end," however, maintains the hyperintense MR signature of on-going inflammation, and continues to expand. Scale bars: (A-G) 1 mm, (H,I) $500 \mu \mathrm{m}, \mathbf{( J )} 200 \mu \mathrm{m}$, (K) $500 \mu \mathrm{m}, \mathbf{( L )} 50 \mu \mathrm{m}, \mathbf{( M , N )} 500 \mu \mathrm{m}$, and (O) $50 \mu \mathrm{m}$.

concentrations and concentrations normalized to total creatine yield the same main results.

Analysis of the dynamic changes between 1 and 3 months were also repeated with regression analysis including white matter (WM) fraction as covariate in repeated measures ANCOVA to ensure that the observed changes were not due to the changes in voxel WM content. WM has higher Ins content than gray matter. WM content was obtained by manually outlining the portion of external capsule that overlapped the MRS voxel volume. The results were found to be independent of the WM volume fraction.

Aging effect was taken into account by adding a group of naïve 8.5 months old rats to the study. They were age-matched control for the 6 months post-injury time point and thus depicted the level of Ins in healthy, aged cortex. It has been shown that the aging lead to elevated astroglia markers on the cortex (Harris et al., 2014, 2015). 


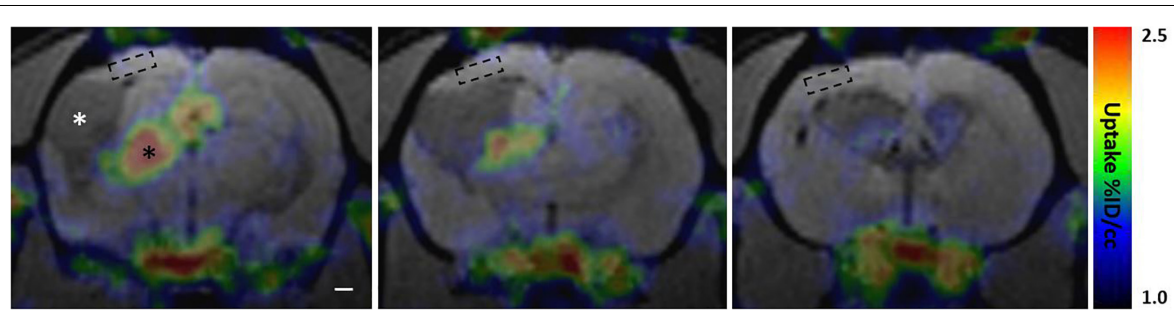

FIGURE 8 | Perilesional cortex and glial envelope show no evident [ $\left.{ }^{18} \mathrm{~F}\right]$-FEPPA binding indicating lack of translocator protein $18 \mathrm{kDa}$ TSPO overexpression. PET signal color map co-localized with MRI (3D-FISP) at three coronal levels of a representative trauma rat 6 weeks post-injury. $\left.{ }^{18} \mathrm{~F}\right]-\mathrm{FEPPA}-\mathrm{PET}$ shows evident TSPO expression in ipsilateral thalamus (black asterisk) while perilesional cortex shows no evident binding of the tracer. In particular, the location of MRS voxel (dashed box) shows no detectable $\left[{ }^{18} \mathrm{~F}\right.$-FEPPA signal, suggesting absence of reactive pro-inflammatory micro- and astroglia. Resting glia or anti-inflammatory glia does not overexpress TSPO, and thus, is not detected in the PET scan. Cerebrospinal-fluid filled lesion cavity is indicated by white asterisk. Glia envelope around the lesion is also expressing only negligible amounts of TSPO appearing void of any $\left[{ }^{18} \mathrm{~F}\right]$-FEPPA signal. Same animal did show marked $\left[{ }^{18} \mathrm{~F}\right.$-FEPPA signal over the lesion and the perilesional cortex in earlier time point, 2 weeks after the injury, but by 6 weeks the signal is gone (Supplementary Figure $\mathbf{3}$ shows the evolvement of $\left[{ }^{18} \mathrm{~F}\right]$-FEPPA signal over time along with T2w images). Rostral tip of the lesion (last image) shows no TSPO expression despite on-going growth. Note that the MRI characteristics of the lesion size, lesion surroundings, and overall atrophy are comparable to those shown in Figure $\mathbf{7}$.

\section{Chronically Elevated Myo-Inositol May Reflect Cortical Plasticity Beyond the Gliosis}

Myo-inositol is a marker of all glia: both the reactive gliosis or the stable astroglia scar. It has been recognized as a marker for inflammation (Harris et al., 2015) but it has also multiple other roles (Duarte et al., 2012). Ins concentrations have been positively correlated with GFAP and S100b positive cell densities in hippocampus of injured rats in other preclinical brain insult models (Filibian et al., 2012; Pascente et al., 2016). It is also found to be elevated in CCI model in the primary injury site 14 days post-injury (Harris et al., 2012) and in lateral FPI model in ipsilateral hippocampus at 5 months post-LFPI (Immonen et al., 2009b). We found elevated concentrations of Ins in all TBI animals at 1 month post-injury and group average remained elevated from 1 to 6 months post-injury despite dual dynamics: "attenuating" or "on-going." The MRS voxel avoided the areas of dense GFAP staining close to the lesion border or along subcortical WM. Importantly, in the context of chronic perilesional cortex that undergoes many forms of plastic remodeling as a delayed response to the injury, the increases in Ins concentration may also associate with the demand for synthesis of inositol-containing phospholipids during synaptogenesis, axonal growth and myelination, as has been suggested in developing brain (Tkac et al., 2003; Kulak et al., 2010; Duarte et al., 2012).

\section{Delayed Increase in Total Choline Suggests Higher Membrane Turnover 6 Months Post-injury Than 1 Month Post-injury}

Increase in total choline (GPC+PCho) is an indicator of membrane turnover (degradation or proliferation) and its elevation in white matter has been used as a marker of diffuse axonal injury and inflammation (Holshouser et al., 2005; Babikian et al., 2006; Shutter et al., 2006; Govind et al., 2010). Choline has been found to be elevated at the primary injury site and surrounding tissue 14 days post-CCI (Harris et al., 2012). Interestingly, our data shows that GPC+PCho in healthy appearing perilesional cortex is not elevated yet at 1 month but becomes elevated 3-6 months post-injury, while Ins is elevated already at 1 month time point. This suggests choline elevation to be associated with membrane turnover processes unrelated to gliosis. These exactly same cortical areas have been shown to undergo number of degradative and regenerative plastic changes in vasculature, extracellular matrix, cellular content, GABAergic interneurons, axonal integrity and connectivity, myelin content, etc. (Avramescu and Timofeev, 2008; Hayward et al., 2010; Cantu et al., 2015; Lehto et al., 2017; Ndode-Ekane et al., 2018; Pitkanen et al., 2018). However, these phenomena should already show at 1 month MRS, while the late increase of choline suggests more delayed processes to take place. Chronically the cortex gets thinner and "squeezed," thus apparently loses some of its extracellular space. While being a complicating factor in the analysis of the absolute concentrations, this phenomenon may also be reflected in the total choline concentration.

\section{High Levels of Antioxidant Glutathione Suggest Sustained Oxidative Stress 6 Months Post-injury}

Glutathione (GSH) is an antioxidant secreted by astrocytes to battle the reactive oxygen species produced by neurons under inflammatory conditions. We found elevated GSH levels in the ipsilateral cortex 1-6 months after TBI while glia marker Ins levels were elevated as well. Increase in GSH levels has been reported in injured rat hippocampus during epileptogenesis after status epilepticus (Filibian et al., 2012) and in cingulate of mild cognitive impairment patients (Duffy et al., 2014). Treatments against oxidative stress have been recently demonstrated to alleviate several disorders in preclinical studies of different brain insults (Pauletti et al., 2017; Frigerio et al., 2018). On the contrary, cortical GSH has been found to be depleted in patients with brain disorders, such as Alzheimer's, mild cognitive impairment, multiple sclerosis, repeated concussions in retired rugby players, as well as upon aging (Benzi et al., 1989; Geremia et al., 1990; 
Choi et al., 2011; Emir et al., 2011; Mandal et al., 2015; Chiang et al., 2017; Gardner et al., 2017). Also iron-mediated inflammation causes depletion of GSH. Decreased levels of blood glutathione peroxidase in chronic phase after severe TBI has been associated with greater disability (Licastro et al., 2016). In our study, the GSH elevation in perilesional cortex months after injury indicates that the brain's ability to combat oxidative stress is increased. GSH levels may increase when astrocytes are chronically activated and recruited, and we observed sustained high concentrations of both Ins and GSH in chronic TBI animals. This suggests chronically sustained, elevated perilesional astrocytic activity, compensatory increase of GSH production due to the sustained battle against oxidative stress, or low GSH depletion. Elevated antioxidant GSH concentration may also associate with the fact that the surviving cortical tissue has been shown to exhibit spontaneous epileptiform activity in a subset of animals in FPI (Reid et al., 2016) and CCI (Yang et al., 2010) models.

\section{$\left[{ }^{18} \mathrm{~F}\right]$-FEPPA-PET Binds to TSPO Overexpressing Reactive Microglia in Thalamus, but Shows No Prominent Binding in Cortex After 1.5 Month Post-injury}

Translocator protein $18 \mathrm{kDa}$ (TSPO), also known as peripheral benzodiazepine receptor (PBR), targeting radiotracers are the largest group of PET tracers for neuroinflammation, and $\left[{ }^{18} \mathrm{~F}\right]$ FEPPA is one of the new generation TSPO tracers with improved binding efficacy (Wilson et al., 2008; Vignal et al., 2018). In a supplementary group of animals $(N=3)$ we saw clear TSPO expression in contusion site at acute stage post-injury 2-4 weeks post-injury, while the cortical $\left[{ }^{18} \mathrm{~F}\right]$-FEPPA signal was abolished by 6-8 weeks post-injury PET scans in the same animals. This is in line with experimental TBI studies utilizing TSPO-PET tracers that have shown the expression to peak 6-7 days postinjury in the injured cortex due to the reactive microgliosis response, stay elevated at 10 days due to reactive astroglia at and around the contusion, and decay by 14-28 days postinjury (Yu et al., 2010; Wang et al., 2014; Israel et al., 2016; Missault et al., 2018). Subacute TSPO expression along the edges of the CCI lesion was recently shown to be elevated 7 days post-injury and returning close to the control level by day 21 post-injury. Interestingly however, the TSPO expression along the lesion edges at day 21 correlated with the long-term outcome in pentylenetetrazol-evoked seizure susceptibility and anxiety in open field test (Missault et al., 2018). Only one study utilizing $\left[{ }^{18} \mathrm{~F}\right]$-FE-DAA1106 after lateral FPI has reported in vivo observable signal up to 9 weeks post-injury in the injured cortex. In the same study, the autoradiography demonstrated TSPO binding along subcortical white matter and along the edges of the cortical lesion to be detectable up to 3 months post-injury (Yu et al., 2010). At this late stage the origin of the signal were a subset of GFAP expressing cells (astroglia) and not the Iba1 positive (microglia) cells (Yu et al., 2010; Wang et al., 2014). Importantly, the perilesional cortex distant from the immediate lesion edge appeared free of TSPO overexpression. $\left[{ }^{18} \mathrm{~F}\right]-\mathrm{FEPPA}$
PET is highly sensitive in detecting pro-inflammatory reactive microgliosis, and in line with literature, we observe strong binding in injured thalamus. Sustained thalamic inflammation and associated reactive gliosis is well documented in this animal model (Pierce et al., 1998; Lehto et al., 2012), and in human TBI (Papadopoulos and Lecanu, 2009; Ramlackhansingh et al., 2011; Coughlin et al., 2015; Faden et al., 2016). However, it is broadly recognized that the TSPO tracers have their limitations as inflammation markers: TSPO is not overexpressed in all subtypes of reactive glia, nor is it expressed in a "stable" astroglia scar (Virdee et al., 2012; Wu et al., 2013; Amhaoul et al., 2014; Tronel et al., 2017). Recent intriguing findings of Beckers et al. (2018), demonstrate that TSPO expression depends on the microglia polarization. TSPO expression was selectively increased in proinflammatory (classically activated M1 type) microglia but not in anti-inflammatory (alternatively activated M2 type) microglia, with activation evoked by IL-4 injection (Beckers et al., 2018). Thus, our preliminary observations of perilesional cortex being TSPO-negative do not suggest that the reactive micro-or astroglia would be absent in chronic stage post-injury, since the presence of them is well documented (Ndode-Ekane et al., 2018) but rather that their activation state has shifted to anti-inflammatory (M2). However, many factors of the TSPO expression in different disease conditions are still unknown.

$\left[{ }^{18} \mathrm{~F}\right]$-FEPPA-negativity of the perilesional cortex indicates that the elevated myo-inositol levels in MRS do not originate from the TSPO expressing glia types, but others. Therefore, we speculate that the mismatch between myo-inositol and TSPO ("Ins-TSPO mismatch" i.e., myo-inositol concentrations being elevated but TSPO-PET being negative) could potentially identify the areas of M2 type glial activity, e.g., on-going plasticity or ongoing glia scar built up of post-injury cortex. Furthermore, the results suggest that the cause for elevated antioxidant GSH levels 3-6 months after TBI are not the reactive oxygen species resulting from local M1 type of microglial response.

\section{Structural Endophenotypes, With Characteristic Speed of Cavity Formation and Glia Scar Maturation, Reflect the Efficacy of the Immunodefence}

The existence of different structural endophenotypes (focal and cavity forming) of lateral FPI rats is a fact that we have been observing in several of our cohorts, and obviously the spectrum of the different lesion types spans the sizes between these two ends at some point of the lesion expansion. Here, we determined the endophenotypes at 1 month post-injury, corresponding a clinically relevant time point for delayed therapies, when the differences in the lesion progression rate can already be seen. Structural endophenotyping is informative as itself. In cavity forming endopenotype the heavy inflammatory response occurs fast, and tight, thin glia scar is formed, while in the focal-cases the response is slower or less efficient and glia scar is thick and diffuse. This suggests that the speed of cavity formation may serve as one indicator of immunodefence efficacy. Moreover, the fact that the proportional growth between 1 and 3 months is largely seized in 
cavity forming cases while is still $115 \%$ in focal cases and $26 \%$ in intermediate cases may have major impact in case-selection of neuroprotective trials.

\section{On-Going Inflammation and Long Lasting Lesion Growth in the Rostral Tip of the Lesion}

The systematic observation throughout the TBI cohort was that in the chronic phase the dorso-rostral edge of the cortical lesion was the site of on-going lesion expansion, immature glia scar and on-going inflammation. In MRI the rostral lesion end was always associated with hematomas, iron residues and often with the T2w signature of on-going inflammation. In endpoint histology the glia envelope of the lesion cavity was wide and disperse at this rostral location while fulfilling the characteristics of and mature, tight glia scar elsewhere. This could indicate that the rostral perilesional cortex is more vulnerable, fails to create the glia shield or have sustained plasticity that hinder the glia envelope formation. The dorsal perilesional tissue also suffers from severe hypoperfusion as well as vascular abnormalities (Hayward et al., 2010), which may predispose it to any further stressors or prevent it from forming the mature glia scar. Certainly, the results imply that the cortical tissue rostrally is not in a stable state, but dealing with infiltrating leukocytes and related stress factors month after month, possibly leading to a local dysfunction. Interestingly, this particular location has also been indicated as putative seizure onset zone in lateral FPI rat model of posttraumatic epilepsy (Reid et al., 2016).

\section{CONCLUSION}

There is a need for in vivo imaging approaches that could assess the putatively sustained or chronically evoked cortical inflammation after TBI. Current scientific consensus is that central nervous system inflammation is one factor determining the long-term outcome and that it offers a promising target for therapies - even late onset treatments. Both the proinflammatory and anti-inflammatory glial activity have disease modifying effects, and discerning the two is a major challenge. Lateral FPI rat model is widely utilized in pre-clinical drug research, and identifying the animals suffering from chronically on-going cortical inflammation will aid to both enrich the population in pre-clinical trials as well as tailor optimally timed therapies. Robust, fast T2w acquisitions are suitable for screening purposes since they are rich in structural information and detect the types of inflammation that attract edema. Evaluating the maturation stage of the glia envelope of the lesion by its T2w MRI properties sheds light in to the disease mechanisms and the sustained plasticity in the lesion edges. Yet, subtle neuroinflammation remains undetected by structural MRI. We found inflammatory MRS profile of myo-inositol, glutathione and choline to be sensitive enough to detect inflammation in MRI-negative cortex. The dynamics of the MRS markers did not go hand-in-hand with the lesion growth rate suggesting independent inflammatory processes along the lesion edge versus further in the cortex. Observations with the third imaging approach, $\left[{ }^{18} \mathrm{~F}\right]$-FEPPA-PET, suggest that TSPO overexpression, that occurs in pro-inflammatory subtype of reactive microglia, may not be present in chronic posttraumatic cortex. Instead, the follow-up of the TSPO expression might serve in indicating the shift in microglial polarization from pro-inflammatory to antiinflammatory state in injured cortex. This is crucial information when planning the imaging paradigm and timing of antiinflammatory treatment trials. Taken together, when probing different aspects of posttraumatic inflammatory processes the MRI, MRS, and PET clearly complement each other.

\section{DATA AVAILABILITY}

The datasets generated for this study are available on request to the corresponding author.

\section{ETHICS STATEMENT}

All animal procedures were approved by the Animal Care and use Committee of the University of Eastern Finland and performed in accordance with the guidelines of European Community Council Directives 2010/63/EU and 86/609/EEC.

\section{AUTHOR CONTRIBUTIONS}

RI, OG, and AP conceived and designed the study. OG and AP provided the resources and research facilities. AY and RI collected and analyzed the MRI and MRS data, and analyzed the histology. PP produced the PET radioligand. KJ conducted the PET measurements. AY, RI, and AP wrote the manuscript. KJ, PP, and $O G$ wrote the sections of the manuscript. All authors revised the manuscript, and read and approved the submitted version.

\section{FUNDING}

This study was supported by the Finnish Cultural Foundation, both Central Fund and North Savo Regional Fund (RI and AY), the Epilepsy Research Foundation (AY), the European Regional Development Fund project A71429 (OG, KJ, and PP), the Medical Research Council of the Academy of Finland [Grants 272249 and 273909 (AP) and Grant 298007 (OG)], and the European Union's Seventh Framework Programme (FP7/20072013) under grant agreement no. 602102 (EPITARGET) (AP).

\section{ACKNOWLEDGMENTS}

We thank Mr. Jarmo Hartikainen and Mrs. Maarit Pulkkinen for their expert technical assistance.

\section{SUPPLEMENTARY MATERIAL}

The Supplementary Material for this article can be found online at: https://www.frontiersin.org/articles/10.3389/fnins.2019. 00863/full\#supplementary-material 


\section{REFERENCES}

Ali, I., Silva, J. C., Liu, S., Shultz, S. R., Kwan, P., Jones, N. C., et al. (2018). Targeting neurodegeneration to prevent post-traumatic epilepsy. Neurobiol. Dis. 123, 100-109. doi: 10.1016/j.nbd.2018.08.006

Amhaoul, H., Staelens, S., and Dedeurwaerdere, S. (2014). Imaging brain inflammation in epilepsy. Neuroscience 279, 238-252. doi: 10.1016/j. neuroscience.2014.08.044

Avramescu, S., and Timofeev, I. (2008). Synaptic strength modulation after cortical trauma: a role in epileptogenesis. J. Neurosci. 28, 6760-6772. doi: 10.1523/ JNEUROSCI.0643-08.2008

Babikian, T., Freier, M. C., Ashwal, S., Riggs, M. L., Burley, T., and Holshouser, B. A. (2006). MR spectroscopy: predicting long-term neuropsychological outcome following pediatric TBI. J. Magn. Reson. Imaging 24, 801-811. doi: 10.1002/ jmri.20696

Beckers, L., Ory, D., Geric, I., Declercq, L., Koole, M., Kassiou, M., et al. (2018). Increased expression of translocator protein (TSPO) marks pro-inflammatory microglia but does not predict neurodegeneration. Mol. Imaging Biol. 20, 94-102. doi: 10.1007/s11307-017-1099-1

Benzi, G., Pastoris, O., Marzatico, F., and Villa, R. F. (1989). Cerebral enzyme antioxidant system. Influence of aging and phosphatidylcholine. J. Cereb. Blood Flow Metab. 9, 373-380. doi: 10.1038/jcbfm.1989.56

Burda, J. E., Bernstein, A. M., and Sofroniew, M. V. (2015). Astrocyte roles in traumatic brain injury. Exp. Neurol. 275(Pt 3), 305-315. doi: 10.1016/j. expneurol.2015.03.020

Cantu, D., Walker, K., Andresen, L., Taylor-Weiner, A., Hampton, D., Tesco, G., et al. (2015). Traumatic brain injury increases cortical glutamate network activity by compromising GABAergic control. Cereb. Cortex 25, 2306-2320. doi: $10.1093 /$ cercor/bhu041

CDC, (2017). Rates of TBI-related Emergency Department Visits, Hospitalizations, and Deaths - United States, 2001 - 2010. Washington, DC: Centers for Disease Control and Prevention.

Chen, S., Pickard, J. D., and Harris, N. G. (2003). Time course of cellular pathology after controlled cortical impact injury. Exp. Neurol. 182, 87-102. doi: 10.1016/ s0014-4886(03)00002-5

Chiang, G. C., Mao, X., Kang, G., Chang, E., Pandya, S., Vallabhajosula, S., et al. (2017). Relationships among cortical glutathione levels, brain amyloidosis, and memory in healthy older adults investigated In Vivo with (1)H-MRS and pittsburgh compound-B PET. AJNR Am. J. Neuroradiol. 38, 1130-1137. doi: 10.3174/ajnr.a5143

Choi, I. Y., Lee, S. P., Denney, D. R., and Lynch, S. G. (2011). Lower levels of glutathione in the brains of secondary progressive multiple sclerosis patients measured by $1 \mathrm{H}$ magnetic resonance chemical shift imaging at $3 \mathrm{~T}$. Mult. Scler. 17, 289-296. doi: 10.1177/1352458510384010

Corps, K. N., Roth, T. L., and McGavern, D. B. (2015). Inflammation and neuroprotection in traumatic brain injury. JAMA Neurol. 72, 355-362. doi: 10.1001/jamaneurol.2014.3558

Coughlin, J. M., Wang, Y., Munro, C. A., Ma, S., Yue, C., Chen, S., et al. (2015). Neuroinflammation and brain atrophy in former NFL players: an in vivo multimodal imaging pilot study. Neurobiol. Dis. 74, 58-65. doi: 10.1016/j.nbd. 2014.10.019

Dadas, A., and Janigro, D. (2018). Breakdown of blood brain barrier as a mechanism of post-traumatic epilepsy. Neurobiol. Dis. 123, 20-26. doi: 10.1016/ j.nbd.2018.06.022

Duarte, J. M., Lei, H., Mlynarik, V., and Gruetter, R. (2012). The neurochemical profile quantified by in vivo $1 \mathrm{H}$ NMR spectroscopy. Neuroimage 61, 342-362. doi: 10.1016/j.neuroimage.2011.12.038

Duffy, S. L., Lagopoulos, J., Hickie, I. B., Diamond, K., Graeber, M. B., Lewis, S. J., et al. (2014). Glutathione relates to neuropsychological functioning in mild cognitive impairment. Alzheimers Dement. 10, 67-75. doi: 10.1016/j.jalz.2013. 01.005

Emir, U. E., Raatz, S., McPherson, S., Hodges, J. S., Torkelson, C., Tawfik, P., et al. (2011). Noninvasive quantification of ascorbate and glutathione concentration in the elderly human brain. NMR Biomed. 24, 888-894. doi: 10.1002/nbm.1646

Faden, A. I., Wu, J., Stoica, B. A., and Loane, D. J. (2016). Progressive inflammationmediated neurodegeneration after traumatic brain or spinal cord injury. Br. J. Pharmacol. 173, 681-691. doi: 10.1111/bph.13179
Filibian, M., Frasca, A., Maggioni, D., Micotti, E., Vezzani, A., and Ravizza, T. (2012). In vivo imaging of glia activation using $1 \mathrm{H}$-magnetic resonance spectroscopy to detect putative biomarkers of tissue epileptogenicity. Epilepsia 53, 1907-1916. doi: 10.1111/j.1528-1167.2012.03685.x

Frigerio, F., Pasqualini, G., Craparotta, I., Marchini, S., van Vliet, E. A., Foerch, P., et al. (2018). n-3 Docosapentaenoic acid-derived protectin D1 promotes resolution of neuroinflammation and arrests epileptogenesis. Brain 141, 3130-3143. doi: 10.1093/brain/awy247

Gardner, A. J., Iverson, G. L., Wojtowicz, M., Levi, C. R., Kay-Lambkin, F., Schofield, P. W., et al. (2017). MR spectroscopy findings in retired professional rugby league players. Int. J. Sports Med. 38, 241-252. doi: 10.1055/s-0042120843

Geremia, E., Baratta, D., Zafarana, S., Giordano, R., Pinizzotto, M. R., La Rosa, M. G., et al. (1990). Antioxidant enzymatic systems in neuronal and glial cellenriched fractions of rat brain during aging. Neurochem. Res. 15, 719-723. doi: 10.1007/bf00973653

Govind, V., Gold, S., Kaliannan, K., Saigal, G., Falcone, S., Arheart, K. L., et al. (2010). Whole-brain proton MR spectroscopic imaging of mild-tomoderate traumatic brain injury and correlation with neuropsychological deficits. J. Neurotrauma 27, 483-496. doi: 10.1089/neu.2009.1159

Harris, J. L., Choi, I. Y., and Brooks, W. M. (2015). Probing astrocyte metabolism in vivo: proton magnetic resonance spectroscopy in the injured and aging brain. Front. Aging Neurosci. 7:202. doi: 10.3389/fnagi.2015.00202

Harris, J. L., Yeh, H. W., Choi, I. Y., Lee, P., Berman, N. E., Swerdlow, R. H., et al. (2012). Altered neurochemical profile after traumatic brain injury: (1)HMRS biomarkers of pathological mechanisms. J. Cereb. Blood Flow Metab. 32, 2122-2134. doi: 10.1038/jcbfm.2012.114

Harris, J. L., Yeh, H. W., Swerdlow, R. H., Choi, I. Y., Lee, P., and Brooks, W. M. (2014). High-field proton magnetic resonance spectroscopy reveals metabolic effects of normal brain aging. Neurobiol. Aging 35, 1686-1694. doi: 10.1016/j. neurobiolaging.2014.01.018

Hayward, N. M., Immonen, R., Tuunanen, P. I., Ndode-Ekane, X. E., Grohn, O., and Pitkanen, A. (2010). Association of chronic vascular changes with functional outcome after traumatic brain injury in rats. J. Neurotrauma 27, 2203-2219. doi: 10.1089/neu.2010.1448

Hayward, N. M., Tuunanen, P. I., Immonen, R., Ndode-Ekane, X. E., Pitkanen, A., and Grohn, O. (2011). Magnetic resonance imaging of regional hemodynamic and cerebrovascular recovery after lateral fluid-percussion brain injury in rats. J. Cereb. Blood Flow Metab. 31, 166-177. doi: 10.1038/jcbfm.2010.67

Hinson, H. E., Rowell, S., and Schreiber, M. (2015). Clinical evidence of inflammation driving secondary brain injury: a systematic review. J. Trauma Acute Care Surg. 78, 184-191. doi: 10.1097/TA.0000000000000468

Holshouser, B. A., Tong, K. A., and Ashwal, S. (2005). Proton MR spectroscopic imaging depicts diffuse axonal injury in children with traumatic brain injury. AJNR Am. J. Neuroradiol. 26, 1276-1285.

Immonen, R. J., Kharatishvili, I., Grohn, H., Pitkanen, A., and Grohn, O. H. (2009a). Quantitative MRI predicts long-term structural and functional outcome after experimental traumatic brain injury. Neuroimage 45, 1-9. doi: 10.1016/j.neuroimage.2008.11.022

Immonen, R. J., Kharatishvili, I., Niskanen, J. P., Grohn, H., Pitkanen, A., and Grohn, O. H. (2009b). Distinct MRI pattern in lesional and perilesional area after traumatic brain injury in rat-11 months follow-up. Exp. Neurol. 215, 29-40. doi: 10.1016/j.expneurol.2008.09.009

Israel, I., Ohsiek, A., Al-Momani, E., Albert-Weissenberger, C., Stetter, C., Mencl, S., et al. (2016). Combined [(18)F]DPA-714 micro-positron emission tomography and autoradiography imaging of microglia activation after closed head injury in mice. J. Neuroinflammation 13:140. doi: 10.1186/s12974-0160604-9

Jullienne, A., Obenaus, A., Ichkova, A., Savona-Baron, C., Pearce, W. J., and Badaut, J. (2016). Chronic cerebrovascular dysfunction after traumatic brain injury. J. Neurosci. Res. 94, 609-622. doi: 10.1002/jnr.23732

Kharatishvili, I., Immonen, R., Grohn, O., and Pitkanen, A. (2007). Quantitative diffusion MRI of hippocampus as a surrogate marker for post-traumatic epileptogenesis. Brain 130, 3155-3168. doi: 10.1093/brain/awm268

Kharatishvili, I., Nissinen, J. P., McIntosh, T. K., and Pitkanen, A. (2006). A model of posttraumatic epilepsy induced by lateral fluid-percussion brain injury in rats. Neuroscience 140,685-697. doi: 10.1016/j.neuroscience.2006.03.012 
Kokiko-Cochran, O. N., and Godbout, J. P. (2018). The inflammatory continuum of traumatic brain injury and Alzheimer's disease. Front. Immunol. 9:672. doi: 10.3389/fimmu.2018.00672

Kulak, A., Duarte, J. M., Do, K. Q., and Gruetter, R. (2010). Neurochemical profile of the developing mouse cortex determined by in vivo $1 \mathrm{H}$ NMR spectroscopy at $14.1 \mathrm{~T}$ and the effect of recurrent anaesthesia. J. Neurochem. 115, 1466-1477. doi: 10.1111/j.1471-4159.2010.07051.x

Kyyriainen, J., Ekolle Ndode-Ekane, X., and Pitkanen, A. (2017). Dynamics of PDGFRbeta expression in different cell types after brain injury. Glia 65, 322-341. doi: 10.1002/glia.23094

Lehto, L. J., Sierra, A., Corum, C. A., Zhang, J., Idiyatullin, D., Pitkanen, A., et al. (2012). Detection of calcifications in vivo and ex vivo after brain injury in rat using SWIFT. Neuroimage 61, 761-772. doi: 10.1016/j.neuroimage.2012.03.002

Lehto, L. J., Sierra, A., and Grohn, O. (2017). Magnetization transfer SWIFT MRI consistently detects histologically verified myelin loss in the thalamocortical pathway after a traumatic brain injury in rat. NMR Biomed. 30:e3678. doi: $10.1002 / \mathrm{nbm} .3678$

Lescot, T., Fulla-Oller, L., Po, C., Chen, X. R., Puybasset, L., Gillet, B., et al. (2010). Temporal and regional changes after focal traumatic brain injury. J. Neurotrauma 27, 85-94. doi: 10.1089/neu.2009.0982

Lewis, D. A., Campbell, M. J., and Morrison, J. H. (1986). An immunohistochemical characterization of somatostatin-28 and somatostatin281-12 in monkey prefrontal cortex. J. Comp. Neurol. 248, 1-18. doi: 10.1002/cne.902480102

Li, W., Long, J. A., Watts, L., Shen, Q., Liu, Y., Jiang, Z., et al. (2016). Spatiotemporal changes in diffusion, T2 and susceptibility of white matter following mild traumatic brain injury. NMR Biomed. 29, 896-903. doi: 10.1002/nbm.3536

Licastro, F., Hrelia, S., Porcellini, E., Malaguti, M., Di Stefano, C., Angeloni, C., et al. (2016). Peripheral inflammatory markers and antioxidant response during the post-acute and chronic phase after severe traumatic brain injury. Front. Neurol. 7:189. doi: 10.3389/fneur.2016.00189

Liu, S., Grigoryan, M. M., Vasilevko, V., Sumbria, R. K., Paganini-Hill, A., Cribbs, D. H., et al. (2014). Comparative analysis of $H \& E$ and prussian blue staining in a mouse model of cerebral microbleeds. J. Histochem. Cytochem. 62, 767-773.

Lucke-Wold, B. P., Nguyen, L., Turner, R. C., Logsdon, A. F., Chen, Y. W., Smith, K. E., et al. (2015). Traumatic brain injury and epilepsy: underlying mechanisms leading to seizure. Seizure 33, 13-23. doi: 10.1016/j.seizure.2015.10.002

Mandal, P. K., Saharan, S., Tripathi, M., and Murari, G. (2015). Brain glutathione levels-a novel biomarker for mild cognitive impairment and Alzheimer's disease. Biol. Psychiatry 78, 702-710. doi: 10.1016/j.biopsych.2015.04.005

McIntosh, T. K., Vink, R., Noble, L., Yamakami, I., Fernyak, S., Soares, H., et al. (1989). Traumatic brain injury in the rat: characterization of a lateral fluid-percussion model. Neuroscience 28, 233-244. doi: 10.1016/0306-4522(89) 90247-9

Missault, S., Anckaerts, C., Blockx, I., Deleye, S., Van Dam, D., Barriche, N., et al. (2018). Neuroimaging of subacute brain inflammation and microstructural changes predicts long-term functional outcome after experimental traumatic brain injury. J. Neurotrauma 36, 768-788. doi: 10.1089/neu.2018.5704

Ndode-Ekane, X. E., Matthiesen, L., Banuelos-Cabrera, I., Palminha, C. A. P., and Pitkanen, A. (2018). T-cell infiltration into the perilesional cortex is long-lasting and associates with poor somatomotor recovery after experimental traumatic brain injury. Restor. Neurol. Neurosci. 36, 485-501. doi: 10.3233/RNN170811

Niskanen, J. P., Airaksinen, A. M., Sierra, A., Huttunen, J. K., Nissinen, J., Karjalainen, P. A., et al. (2013). Monitoring functional impairment and recovery after traumatic brain injury in rats by FMRI. J. Neurotrauma 30, 546-556. doi: 10.1089/neu.2012.2416

Nissinen, J., Andrade, P., Natunen, T., Hiltunen, M., Malm, T., Kanninen, K., et al. (2017). Disease-modifying effect of atipamezole in a model of post-traumatic epilepsy. Epilepsy Res. 136, 18-34. doi: 10.1016/j.eplepsyres.2017.07.005

Papadopoulos, V., and Lecanu, L. (2009). Translocator protein (18 kDa) TSPO: an emerging therapeutic target in neurotrauma. Exp. Neurol. 219, 53-57. doi: 10.1016/j.expneurol.2009.04.016

Pascente, R., Frigerio, F., Rizzi, M., Porcu, L., Boido, M., Davids, J., et al. (2016). Cognitive deficits and brain myo-Inositol are early biomarkers of epileptogenesis in a rat model of epilepsy. Neurobiol. Dis. 93, 146-155. doi: 10.1016/j.nbd.2016.05.001
Pauletti, A., Terrone, G., Shekh-Ahmad, T., Salamone, A., Ravizza, T., Rizzi, M., et al. (2017). Targeting oxidative stress improves disease outcomes in a rat model of acquired epilepsy. Brain 140, 1885-1899. doi: 10.1093/brain/awx117

Paxinos, G., and Watson, C. (1998). The Rat Brain in Stereotaxic Coordinates. London: Academic Press.

Pearn, M. L., Niesman, I. R., Egawa, J., Sawada, A., Almenar-Queralt, A., Shah, S. B., et al. (2017). Pathophysiology associated with traumatic brain injury: current treatments and potential novel therapeutics. Cell Mol. Neurobiol. 37, 571-585. doi: 10.1007/s10571-016-0400-1

Pierce, J. E., Smith, D. H., Trojanowski, J. Q., and McIntosh, T. K. (1998). Enduring cognitive, neurobehavioral and histopathological changes persist for up to one year following severe experimental brain injury in rats. Neuroscience 87 , 359-369. doi: 10.1016/s0306-4522(98)00142-0

Pitkanen, A., Ekolle Ndode-Ekane, X., Lapinlampi, N., and Puhakka, N. (2018). Epilepsy biomarkers - Toward etiology and pathology specificity. Neurobiol. Dis. 123, 42-58. doi: 10.1016/j.nbd.2018.05.007

Provencher, S. W. (2001). Automatic quantitation of localized in vivo $1 \mathrm{H}$ spectra with LCModel. NMR Biomed. 14, 260-264. doi: 10.1002/nbm.698

Ramlackhansingh, A. F., Brooks, D. J., Greenwood, R. J., Bose, S. K., Turkheimer, F. E., Kinnunen, K. M., et al. (2011). Inflammation after trauma: microglial activation and traumatic brain injury. Ann. Neurol. 70, 374-383. doi: 10.1002/ ana. 22455

Reid, A. Y., Bragin, A., Giza, C. C., Staba, R. J., and Engel, J. (2016). The progression of electrophysiologic abnormalities during epileptogenesis after experimental traumatic brain injury. Epilepsia 57, 1558-1567. doi: 10.1111/epi.13486

Shutter, L., Tong, K. A., Lee, A., and Holshouser, B. A. (2006). Prognostic role of proton magnetic resonance spectroscopy in acute traumatic brain injury. J. Head Trauma Rehabil. 21, 334-349. doi: 10.1097/00001199-20060700000005

Sofroniew, M. V., and Vinters, H. V. (2010). Astrocytes: biology and pathology. Acta Neuropathol. 119, 7-35. doi: 10.1007/s00401-009-0619-8

Sun, M., Brady, R. D., Wright, D. K., Kim, H. A., Zhang, S. R., Sobey, C. G., et al. (2017). Treatment with an interleukin-1 receptor antagonist mitigates neuroinflammation and brain damage after polytrauma. Brain Behav. Immun. 66, 359-371. doi: 10.1016/j.bbi.2017.08.005

Thompson, H. J., Lifshitz, J., Marklund, N., Grady, M. S., Graham, D. I., Hovda, D. A., et al. (2005). Lateral fluid percussion brain injury: a 15-year review and evaluation. J. Neurotrauma 22, 42-75. doi: 10.1089/neu.2005.22.42

Tkac, I., Rao, R., Georgieff, M. K., and Gruetter, R. (2003). Developmental and regional changes in the neurochemical profile of the rat brain determined by in vivo $1 \mathrm{H}$ NMR spectroscopy. Magn. Reson. Med. 50, 24-32. doi: 10.1002/ mrm. 10497

Tkac, I., Starcuk, Z., Choi, I. Y., and Gruetter, R. (1999). In vivo 1H NMR spectroscopy of rat brain at $1 \mathrm{~ms}$ echo time. Magn. Reson. Med. 41, 649-656. doi: 10.1002/(sici)1522-2594(199904)41:4<649::aid-mrm2>3.0.co;2-g

Tronel, C., Largeau, B., Santiago Ribeiro, M. J., Guilloteau, D., Dupont, A. C., and Arlicot, N. (2017). Molecular targets for PET imaging of activated microglia: the current situation and future expectations. Int. J. Mol. Sci. 18:E802. doi: $10.3390 /$ ijms 18040802

van Vliet, E. A., Dedeurwaerdere, S., Cole, A. J., Friedman, A., Koepp, M. J., Potschka, H., et al. (2017). WONOEP appraisal: imaging biomarkers in epilepsy. Epilepsia 58, 315-330. doi: 10.1111/epi.13621

Vignal, N., Cisternino, S., Rizzo-Padoin, N., San, C., Hontonnou, F., Gele, T., et al. (2018). [18F]FEPPA a TSPO radioligand: optimized radiosynthesis and evaluation as a PET radiotracer for brain inflammation in a peripheral LPS-Injected mouse model. Molecules 23:E1375. doi: 10.3390/molecules230 61375

Virdee, K., Cumming, P., Caprioli, D., Jupp, B., Rominger, A., Aigbirhio, F. I., et al. (2012). Applications of positron emission tomography in animal models of neurological and neuropsychiatric disorders. Neurosci. Biobehav. Rev. 36, 1188-1216. doi: 10.1016/j.neubiorev.2012.01.009

Wang, Y., Yue, X., Kiesewetter, D. O., Niu, G., Teng, G., and Chen, X. (2014). PET imaging of neuroinflammation in a rat traumatic brain injury model with radiolabeled TSPO ligand DPA-714. Eur. J. Nucl. Med. Mol. Imaging 41, 1440-1449. doi: 10.1007/s00259-014-2727-5

Wilson, A. A., Garcia, A., Parkes, J., McCormick, P., Stephenson, K. A., Houle, S., et al. (2008). Radiosynthesis and initial evaluation of [18F]-FEPPA for PET 
imaging of peripheral benzodiazepine receptors. Nucl. Med. Biol. 35, 305-314. doi: 10.1016/j.nucmedbio.2007.12.009

Wu, H. M., Huang, S. C., Vespa, P., Hovda, D. A., and Bergsneider, M. (2013). Redefining the pericontusional penumbra following traumatic brain injury: evidence of deteriorating metabolic derangements based on positron emission tomography. J. Neurotrauma 30, 352-360. doi: 10.1089/neu.2012. 2610

Xu, S., Zhuo, J., Racz, J., Shi, D., Roys, S., Fiskum, G., et al. (2011). Early microstructural and metabolic changes following controlled cortical impact injury in rat: a magnetic resonance imaging and spectroscopy study. J. Neurotrauma 28, 2091-2102. doi: 10.1089/neu.2010.1739

Yang, L., Afroz, S., Michelson, H. B., Goodman, J. H., Valsamis, H. A., and Ling, D. S. (2010). Spontaneous epileptiform activity in rat neocortex after controlled cortical impact injury. J. Neurotrauma 27, 1541-1548. doi: 10.1089/neu.2009. 1244

Yoo, R. E., Choi, S. H., Oh, B. M., Do Shin, S., Lee, E. J., Shin, D. J., et al. (2018). Quantitative dynamic contrast-enhanced MR imaging shows widespread blood-brain barrier disruption in mild traumatic brain injury patients with post-concussion syndrome. Eur. Radiol. 29, 1308-1317. doi: 10. 1007/s00330-018-5656-z

Yu, I., Inaji, M., Maeda, J., Okauchi, T., Nariai, T., Ohno, K., et al. (2010). Glial cell-mediated deterioration and repair of the nervous system after traumatic brain injury in a rat model as assessed by positron emission tomography. J. Neurotrauma 27, 1463-1475. doi: 10.1089/neu.2009.1196

Conflict of Interest Statement: The authors declare that the research was conducted in the absence of any commercial or financial relationships that could be construed as a potential conflict of interest.

Copyright () 2019 Yasmin, Pitkänen, Jokivarsi, Poutiainen, Gröhn and Immonen. This is an open-access article distributed under the terms of the Creative Commons Attribution License (CC BY). The use, distribution or reproduction in other forums is permitted, provided the original author(s) and the copyright owner(s) are credited and that the original publication in this journal is cited, in accordance with accepted academic practice. No use, distribution or reproduction is permitted which does not comply with these terms. 